Mathematisches Forschungsinstitut Oberwolfach

Report No. 34/2010

DOI: $10.4171 /$ OWR/2010/34

\title{
Mathematical Theory and Modelling in Atmosphere-Ocean-Science
}

\author{
Organised by \\ Andrew J. Majda, New York \\ Bjorn Stevens, Hamburg \\ Rupert Klein, Berlin
}

August 8th - August 14th, 2010

\begin{abstract}
Participants from around the world gathered to review application and development of mathematics in relation to problems in the atmospheric, oceanic and climate sciences.
\end{abstract} Mathematics Subject Classification (2000): 86A10, 86A06, 76U05, 7606, 65M12, 65Z05, 60G20,
62F15, 35M10.

\section{Introduction by the Organisers}

The workshop Mathematical Theory and Modelling in Atmosphere-Ocean-Science, organized by Andrew J. Majda (New York), Rupert Klein (Berlin) and Bjorn Stevens (Hamburg) was held August 8th-August 14th, 2010. This meeting was the third workshop of its type over the last decade, and has helped to cultivate interests in mathematics as related to the atmospehric, oceanic and climate sciences. This year's workshop was well attended by over 50 participants from mostly North America and Europe. Participants from the broader fields of fluid dynamics, atmospheric, oceanic and climate science were joined by mathematicians with interests in PDEs, stochastics and numerical methods. The workshop touched on a wide range of themes including: (i) data assimilation; (ii) multi-scale asympototics; (iii) numerical methods for PDEs; (iv) stochastic methods; (v) information theory, (vi) time-series analysis; (vii) turbulence; and (viii) geophysical fluid dynamics. As such the workshop outlined the cutting edge of applied mathematics as related to problems in the atmospheric and oceanic sciences. 
In a departure from the standard Oberwolfach workshop, but in keeping with the breadth of the material covered, all participants were invited to give a short presentation outlining critical issues or results pertaining to their research. These short talks, which are sketched out in the abstracts below, served as the basis for more focused discussions among those with relevant expertise and interest. As is evident from the material below, the development and application of mathematics to atmospheric and oceanic science is a vibrant enterprise, and the talks gave participants the chance to see the breadth of the science within the intimate setting that Oberwolfach provides. 


\section{Workshop: Mathematical Theory and Modelling in Atmosphere- Ocean-Science}

\section{Table of Contents}

Ulrich Achatz (joint with Rupert Klein, Fabian Senf)

Gravity waves, scale asymptotics, and the pseudo-incompressible equations

Tommaso Benacchio (joint with L. Bonaventura, MOX-Politecnico di Milano) Spectral collocation methods on semi-infinite domains and application to open boundary conditions

Joseph A. Biello (joint with Andrew J. Majda)

An asymptotic description of the interaction of waves on the ITCZ with

midlatitude quasigeostrophic dynamics .................. 2035

Simona Bordoni (joint with Tapio Schneider)

What drives large-scale monsoons in the Earth's atmosphere?

Yann Brenier

Long time impact of a small, slowly evolving, source term in a convection model

Oliver Bühler (joint with Miranda Holmes-Cerfon)

The rapid decay of large-scale internal tides due to interactions with random small-scale topography .............................. 2040

Paola Cessi (joint with Christopher L. Wolfe)

What sets the oceanic mid-depth stratification and overturning circulation?

George C. Craig

Statistics and interactions in deep cumulus convection

Stamen Iankov Dolaptchiev (joint with Ilya Timofeyev, Ulrich Achatz)

Subgrid scale closure for the Burgers equation based on stochastic mode

reduction ........................................ 2044

Joseph Egger (joint with Klaus-Peter Hoinka, Thomas Spengler)

Is potential temperature inversion complementary to potential vorticity inversion? . . . . . . . . . . . . . . . . . . . . . . . . . . 2044

Jason Frank (joint with Svetlana Dubinkina, Ben Leimkuhler)

Thermostats for point vortices

Christian Franzke (joint with Daniel Peavoy)

Identification of climate switching during the last glacial using Bayesian statistical methods 
Dargan M. W. Frierson (joint with Yen-Ting Hwang, Sarah M. Kang)

Energy transports and tropical precipitation shifts in simulations of 20th and 21st century climate

Edwin P. Gerber

On the time scales of midlatitude atmospheric variability

Dimitrios Giannakis (joint with Andrew J. Majda)

Identifying and predicting the extensional and meandering phases of the

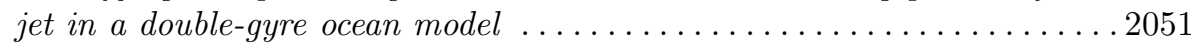

Francis X. Giraldo (joint with James Kelly, Emil Constantinescu)

Development of the non-hydrostatic unified model of the atmosphere

(NUMA)

Andrey Gritsun

Estimation of the sensitivity of atmospheric systems using

fluctuation-dissipation theorem and unstable periodic orbits

Marcus J. Grote (joint with Andrew J. Majda)

Entropy, targeted, and redundant observations for filtering turbulent signals

Illia Horenko

Nonstationarity in discrete multifactor models, memory and application to cloud modeling

Boualem Khouider (joint with Andrew Majda, Amik St-Cyr, Joseph Tribbia) Simulation of the MJO in a coarse resolution GCM using a simple multicloud parametrization

Peter Korn

On A Mimetic Discretization of the Ocean Primitive Equations .......2058

Frank Kwasniok

Stochastic subgrid-scale parametrisation using cluster-weighted modelling 2060

Juan Pedro Mellado (joint with Bjorn Stevens, Heiko Schmidt, Norbert

Peters)

Investigation of latent heat effects at the stratocumulus top using direct numerical simulations ................................2061

Andreas Müller (joint with Jörn Behrens, Francis X. Giraldo, Volkmar Wirth)

An adaptive discontinuous Galerkin method for modelling cumulus

David J. Muraki (joint with Andrea Blazenko, Kevin Mitchell \& Chris Snyder)

A potential vorticity dynamics for rotating shallow water on the sphere .2064

John Norbury (joint with Anthony Lock)

Some exact solutions for a column model of moist convection 
Antony Z. Owinoh (joint with Omar Knio, Rupert Klein)

Flow structure and uncertainty estimation of Ekman boundary layer flow over rough surfaces ..............................2067

Eileen Päschke (joint with Antony Owinoh, Rupert Klein) A generalized Eliassen balanced vortex model

Olivier Pauluis (joint with Frédéric Laliberté and Tiffany Shaw)

Averaging the global atmospheric circulation . . . . . . . . . . . . . . . . 2070

Sebastian Reich (joint with Kay Bergemann, Georg Gottwald)

Data assimilation

Marco Restelli

Hybridizable discontinuous Galerkin methods for geophysical applications 2073

Ian Roulstone

Incompressible Navier-Stokes flows: eigenvalue problems and complex

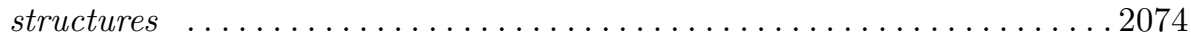

Frank Selten (joint with Leonie van de Berge,Wim Wiegerinck,Gregory Duane)

A multi-model ensemble method that combines imperfect models through learning

Fabian Senf (joint with Ulrich Achatz)

The impact of solar thermal tides on the propagation and dissipation of gravity waves

Leslie Smith (joint with Susan Kurien) Aspect ratio effects in rotating Boussinesq flows . . . . . . . . . . . 2078

Shafer Smith (joint with Elsa Bernard)

Geostrophic turbulence near rapid changes in stratification

Ashwanth Srinivasan (joint with W. C. Thacker, M. Iskandarani, O. M. Knio)

Uncertainty propagation and quantification in numerical ocean simulations using polynomial chaos expansions

Samuel N. Stechmann (joint with Bjorn Stevens)

Multiscale models for cumulus cloud dynamics

Esteban G. Tabak (joint with Paul A. Milewski)

The diurnal cycle and the meridional extent of the tropics

Bruce Turkington (joint with Petr Plecháč)

Coarse-graining of deterministic dynamics via statistical estimation and optimization ..................................2084

Jacques Vanneste (joint with François Lott and Riwal Plougonven)

Inertia-gravity-wave generation by sheared potential-vorticity anomalies 2086 
Xiaoming Wang (joint with Andrew J. Majda)

Linear response theory for statistical ensembles in complex systems with time-periodic forcing

Andreas Will (joint with Jack Ogaja)

Higher order spatial discretisation methods for non-hydrostatic models of the atmosphere on regular grids $\ldots \ldots \ldots \ldots \ldots \ldots \ldots \ldots \ldots \ldots \ldots$

Paul D. Williams

A simple method to improve the time-stepping in atmosphere and ocean models .................................... 2091

William R. Young (joint with Kaushik Srinivasan) Zonation

Vladimir Zeitlin (joint with François Bouchut, Jonathan Gula)

Instabilities of buoyancy driven coastal currents and their nonlinear evolution in the two-layer rotating shallow water model 2094 


\author{
Abstracts \\ Gravity waves, scale asymptotics, and the pseudo-incompressible \\ equations \\ ULRICH ACHATZ \\ (joint work with Rupert Klein, Fabian Senf)
}

Multiple-scale asymptotics is used to analyze the Euler equations for the dynamical situation of a gravity wave (GW) near breaking level. A simple saturation argument in combination with linear theory is used to obtain the relevant dynamical scales. As small expansion parameter the ratio of inverse of the vertical wave number and potential-temperature and pressure scale heights is used, which we allow to be of the same order of magnitude here. It is shown that the resulting equation hierarchy is consistent with that obtained from the pseudo-incompressible equations, both for non-hydrostatic and hydrostatic gravity waves, while this is not the case for the anelastic equations unless the additional assumption of sufficiently weak stratification is adopted. To describe vertical propagation of wave packets over several atmospheric scale heights, WKB theory is used to show that the pseudoincompressible flow divergence generates the same amplitude equation that also obtains from the full Euler equations. This gives a mathematical justification for the use of the pseudo-incompressible equations for studies of gravity-wave breaking in the atmosphere for arbitrary background stratification. The WKB theory interestingly also holds at wave amplitudes close to static instability. In the mean-flow equations we obtain in addition to the classic wave-induced momentum-flux divergences a wave-induced correction of hydrostatic balance in the vertical-momentum equation which cannot be obtained from Boussinesq or anelastic dynamics.

\title{
REFERENCES
}

[1] Achatz, U., Klein, R., and F. Senf Gravity waves, scale asymptotics, and the pseudoincompressible equations, J. Fluid Mech. (2010, in press)

\section{Spectral collocation methods on semi-infinite domains and application to open boundary conditions \\ Tommaso BenacChio \\ (joint work with L. Bonaventura, MOX-Politecnico di Milano)}

The aim of the work is to study the analytical and numerical aspects of a spectral approach to the problem of open boundary conditions, with particular attention to applications in environmental modelling. Indeed, in many situations one may be interested in solutions to differential problems defined on unbounded or semi-infinite domains; in these cases an artificial boundary $\mathcal{B}$ is introduced to encompass a finite region of interest, and the equations are solved in the finite domain by standard numerical discretization techniques [3]. However, on one 
hand, for the problem to be well-posed, suitable boundary conditions have to be enforced on $\mathcal{B}$; on the other hand, no spurious or unphysical phenomena should arise throughout this process. This is for instance the case of vertical propagation of internal gravity waves in the atmosphere: if the upper boundary of the domain is not designed properly, it can give rise to spurious reflection of wave energy back into the computational domain [6]. While conventional boundary conditions such as rigid lid or constant pressure surfaces entail total reflection of wave energy, two classical solutions for this problem, e.g. radiative boundary conditions and absorbing/sponge layers, have been extensively applied in the last three decades to atmospheric models for numerical weather prediction and climate $[2,4,8,5,7]$.

In the context of the present work, we explore the possibility to tackle the problem in a more direct way, approximating the equations of interest with spectral methods in a semi-infinite domain attached to the finite region of interest. To this end, we review the main results concerning polynomial approximation and interpolation on the real positive half line by means of Laguerre polynomials and Laguerre functions, with specific reference to quadrature formulas and spectral derivatives $[9,10]$. Notably, we take into account a special class of generalized Laguerre functions dependent on a parameter $\beta$, tuning which we are able to represent functions on different spatial scales in the same framework [11]. Next, we apply these results to the numerical approximation of Partial Differential Equations with spectral methods on semi-infinite domains. In particular, we consider the advection-diffusion equation and the shallow water equations for open channel flow, discretized on the half line with a pseudospectral (collocation) method and semi-implicit time discretization.

The resulting stable, spectrally accurate method yields a reliable tool which in principle can be interfaced with any numerical discretization of the equations in a finite domain. Among other advantages, the proposed method could outperform the traditional absorbing layer approaches in terms of computational cost, as long as low-order Gaussian quadrature formulas can be used without excessive degradation of the solution accuracy. As a demonstration of the feasibility of this approach, in our work we consider a standard finite volume discretization on the finite domain, attaching to the right endpoint of the interval the spectral discretization on a semi-infinite domain. Numerical simulations show that a reasonably low number of spectral base functions is sufficient to reproduce the solution behaviour at infinity. A more complete description of methods and numerical results can be found in [1].

\section{REFERENCES}

[1] T. Benacchio, Spectral collocation methods on semiinfinite domains and application to open boundary conditions, M.Sc. thesis, MOX-Politecnico di Milano (2010), available at: http://mox.polimi.it/it/progetti/pubblicazioni/tesi/benacchio.pdf.

[2] B. Engquist and A. Majda, Absorbing boundary conditions for the numerical simulation of waves, Mathematics of Computation 31 (1977), 629-651. 
[3] D. Givoli, Computational Absorbing Boundaries, in S. Marburg and B. Nolte, editors, Computational Acoustics of Noise Propagation in Fluids - Finite and Boundary Element Methods (2008), Springer Berlin Heidelberg.

[4] M. Israeli and S.A. Orszag, Approximation of radiation boundary condtions, Journal of Computational Physics, 41 (1981), 115-135.

[5] J.B.Klemp, J. Dudhia and A.D. Hassiotis, An upper gravity-wave absorbing layer for NWP applications, Monthly Weather Review, 136 (2008), 3987-4004.

[6] J.B. Klemp and D.R. Durran, An upper boundary condition permitting internal gravity wave radiation in numerical mesoscale models, Monthly Weather Review, 111 (1983), 430-444.

[7] A. Modave, É. Deleersnijder, and É.J.M. Delhez, On the parameters of absorbing layers for shallow water models, Ocean Dynamics, 60 (2010), 65-79.

[8] P.J. Rasch, Toward atmosphere without tops: Absorbing upper boundary conditions for numerical models, Quarterly Journal of the Royal Meteorological Society, 112 (1986), 11951218.

[9] J. Shen, Stable and efficient spectral methods in unbounded domains using Laguerre functions, SIAM Journal on Numerical Analysis, 38 (2001), 1113-1133.

[10] J.Shen and L.-L. Wang, Some recent advances on spectral methods for unbounded domains, Communications in Computational Physics, 5 (2009), 195-241.

[11] Z.-Q. Wang, B.-Y. Guo, and Y.-N. Wu, Pseudospectral method using generalized Laguerre functions for singular problems on unbounded domains, Discrete and Continuous Dynamical Systems Series B, 11 (2009), 1019-1038.

\section{An asymptotic description of the interaction of waves on the ITCZ with midlatitude quasigeostrophic dynamics

\author{
Joseph A. BIELLO
} \\ (joint work with Andrew J. Majda)}

In a ground breaking paper, Majda \& Klein [1] introduced two new dynamical regimes describing the tropical troposphere. The first regime, called IPESD, describes the modulation of planetary scale flows through the upscale fluxes from equatorial synoptic scale dynamics on intraseasonal timescales. This asymptotic regime provides a framework for the recent multiscale models of the MaddenJulian oscillation $[2,3]$. The second regime, called MEWTG (for mesoscale equatorial weak temperature gradient), describes a circulation forced (primarily) by latent heating due to moist convection. The weak temperature gradient feature of this regime implies that diabatic heating balances vertical velocity on equatorial mesoscales, $O(500 \mathrm{~km})$, and within timescales of less than one day.

Dolaptchiev [4] generalized the model by allowing for stronger temperature variations over larger scales. Majda has recognized that this new multiscale MEWTG is a closed model by going to higher order in the asymptotics [5]. The resulting model contains the original MEWTG equations modulated by a large scale, zonally propagating gravity wave. The temperature and wind properties of the large scale wave are independent of both the zonal and meridional mesoscales and are, thereby, consistent with the weak temperature gradient nature of the tropics.

In my talk I showed that the mean zonal winds described by the multiscale MEWTG equations become unbounded at large latitudes away from the equatorial belt. Unless specific restrictions are made on the mean of the diabatic 
heating, the theory also yields solutions with non-zero meridional velocities far from the equatorial belt; i.e. an open Hadley circulation. Furthermore, meridional geostrophic balance (equivalently, thermal wind balance) is not described by this theory, yet this is known to be the main balance for subtropical and midlatitude winds. These facts strongly suggest that a meridional matching condition must be supplied both to close the multiscale MEWTG theory and to describe the interaction of the equatorial flows with midlatitude dynamics.

MEWTG uses a non-dimensionalization of the equatorial primitive equations where horizontal velocities are measured in units of $5 \mathrm{~m} / \mathrm{s}$; vertical velocity, 5 $\mathrm{cm} / \mathrm{s}$; heating rates, 33 Kelvin/day; temperatures, 3 Kelvin; horizontal scale, 500 $\mathrm{km}$; time scale, 1 day. These equations, with Coriolis parameter extended to the whole sphere, are

$$
\begin{array}{rlrl}
\frac{D}{D t} u-\frac{\sin (\epsilon y) v}{\epsilon}+\frac{\Pi_{x}}{\epsilon} & =S_{u}, & & u_{x}+v_{y}+w_{z}=0 \\
\frac{D}{D t} v+\frac{\sin (\epsilon y) u}{\epsilon}+\frac{\Pi_{y}}{\epsilon} & =S_{v}, & & \Pi_{z}=\Theta \\
\epsilon \frac{D}{D t} \Theta+w & =S_{\theta} &
\end{array}
$$

where the asymptotic parameter $\epsilon \sim 0.1$ is a measure of the weakness of the tropical temperature gradient. We show that multiscale MEWTG arises from (1) at the first two orders of a regular asymptotic expansion of the variables allowing for large zonal variations $(X=\epsilon x)$ and for meridional extents, $y \sim O(1)$. The classical quasigeostrophic balance appropriate to midlatitudes also arises at the first two orders of a regular asymptotic expansion assuming latitudes away from the equator $(\epsilon y \sim O(1))$ and weaker heating rates, $S_{\theta} \sim O(\epsilon)$.

These two dynamical regimes can be connected through a subtropical transition layer with a stretched meridional coordinate, $Y=\epsilon^{1 / 3} y$, and weaker heating, $S \sim O\left(\epsilon^{1 / 3}\right)$. The subtropical asymptotics describe stronger zonal flows and temperature gradients, but meridional flows have the same order of magnitude as those in the deep tropics and midlatitudes

$$
\begin{array}{ll}
u=\epsilon^{-2 / 3}\left(U(X, Y)+\epsilon^{1 / 3} u^{\prime}(X, Y, t)\right) & v=V(X, Y)+v^{\prime}(X, Y, t) \\
\Pi=\epsilon^{-1 / 3}\left(\Pi(X, Y)+\epsilon^{1 / 3} \Pi^{\prime}(X, Y, t)\right) & w=\epsilon^{1 / 3}\left(W(X, Y)+w^{\prime}(X, Y, t)\right) .
\end{array}
$$

The subtropical matching equations break up into a steady circulation and fluctuations. The steady flows

$$
\begin{array}{r}
\left(U \partial_{X}+V \partial_{Y}+W \partial_{z}\right) U-Y V=-d U, \\
Y U+\Pi_{Y}=0, \quad W=\bar{S} \\
U_{X}+V_{Y}+W_{z}=0, \quad \Pi_{z}=\Theta
\end{array}
$$


are in weak temperature gradient and thermal wind balance and the equations describe the zonal variation of the Hadley circulation. The fluctuations,

$$
\begin{aligned}
u_{t}^{\prime}+\left(v^{\prime} \partial_{Y}+w^{\prime} \partial_{z}\right) U-Y v^{\prime}=0 & \\
Y w^{\prime}+\Pi_{Y}^{\prime}=0 & w^{\prime}=S^{\prime} \\
u_{X}^{\prime}+v_{Y}^{\prime}+w_{z}^{\prime}=0 & \Pi_{z}^{\prime}=\Theta^{\prime}
\end{aligned}
$$

conserve total angular momentum. The subtropical meridional velocity and potential temperature are matched to multiscale MEWTG as $Y \rightarrow 0$ and with midlatitude geostrophic dynamics as $Y \rightarrow \infty$.

\section{REFERENCES}

[1] A.J. Majda \& R. Klein, Systematic multiscale models for the tropics, J. Atmos. Sci. 60, 393-408 (2003),

[2] A.J. Majda \& J.A. Biello A new multiscale model for the Madden-Julian oscillation, J. Atmos. Sci, 62, 1694-1721 (2005) .

[3] J.A. Biello \& A.J. Majda Intraseasonal multiscale moist dynamics of the tropical atmosphere, Comm. Math. Sci. 8, 519-540 (2010) .

[4] S. Dolaptchiev, An asymptotic, nonlinear model for anisotropic large scale flows in the tropics., Potsdam Inst. for Climate Report 101, (2005).

[5] A.J. Majda, J.A. Biello \& B. Khouider, Multiscale MEWTG, (in progress).

\section{What drives large-scale monsoons in the Earth's atmosphere?}

\section{Simona Bordoni}

(joint work with Tapio Schneider)

The word monsoon comes from the Arabic word mausim for season [1]. For Arab merchant sailors of the past, the defining aspect of seasons was the reversal of the near-surface winds over the Indian Ocean from the northeast during the winter to the southwest during the summer, in association with what we now understand to be the Asian monsoon. The strong cross-equatorial southwesterly wind, which flows from the coast of Africa to the shores of India and is known as the Somali Jet, is one of the strongest low-level jets in the Earth's atmosphere [2,3]. With increased understanding of monsoon systems, the word monsoon has been more generally applied to the seasonal reversal of both the atmospheric circulation and the associated precipitation patterns over the tropical and subtropical continents, and adjacent oceans, of Asia, Australia, Africa, and both North and South America, which all feature monsoonal climates. Despite the importance of monsoons in the global circulation of the atmosphere and oceans, and their strong impact on society, agriculture and economics around the world, the fundamental mechanisms responsible for monsoons are still not completely understood.

The classic view of monsoons has been based on the existence of a strong contrast in temperature between ocean and land masses, arising from their differing thermal response to the annual cycle of radiative heating $[1,4]$, with monsoons manifesting themselves as planetary-scale sea breeze circulations [5]. However, this depiction of monsoons fails to account for, among other features, the rapidity 
with which changes in precipitation and atmospheric circulation patterns occur at monsoon onset [6].

In this talk, we presented results from simulations with an idealized general circulation model (GCM) coupled to a slab ocean (i.e., in aquaplanet configuration), which show that rapid transitions in the tropical overturning circulation can occur even in the absence of land-sea contrast, provided that the lower boundary has low enough thermal inertia. These transitions are accompanied by precipitation and atmospheric circulation changes similar to those occurring in large-scale monsoon regions in the Earth's atmosphere. Consistent with similar simulations with a dry GCM [7], the rapid transitions in the tropical overturning circulation mark shifts in the dominant balance of the zonal momentum budget in the circulation upper branch, from regimes in which momentum flux divergence by large-scale, midlatitude eddies dominates near the center of the circulation cell, to regimes in which the eddy momentum flux divergence is negligible and the mean momentum flux divergence dominates. Feedbacks among large-scale eddy fluxes, upper-level winds, and the tropical overturning circulation mediate these transitions and render them rapid, if the near-surface moist static energy is able to adjust rapidly, therefore requiring a lower boundary with sufficiently low thermal inertia $[7,6]$. These results therefore suggest that subtropical continents might be needed for monsoon development only insofar as they provide a surface with low enough thermal inertia for the feedback mechanisms between the large-scale eddies and the overturning circulation to be able to act on intraseasonal time scales. Monsoons can occur over an homogeneous swamp surface.

Unlike the global monsoon simulated in the aquaplanet simulations, Earth's monsoons are zonally localized and it is to be expected that surface inhomogeneities, such as land masses and tropography, will modify the dynamical mechanisms described above and will influence the morphology of the monsoonal flow. Future work will be aimed at elucidating the role of purely dynamical mechanisms that act irrespectively of surface inhomogeneities (i.e., the feedbacks between the large-scale extratropical waves and the tropical circulation) and such surface inhomogenieties (i.e., distribution and orography of tropical and subtropical landmasses) in the dynamics of large-scale monsoons.

\section{REFERENCES}

[1] P. J. Webster, The elementary monsoon, in Monsoons. Wiley, New York (1987), 3-32.

[2] J. Findlater, A major low-level air current near the Indian Ocean during the northern summer, Quart. J. R. Met. Soc., 125 (1969), 1527-1550.

[3] T. N. Krishnamurti and H. N. Bhalme, Oscillations of a monsoons system. Part I: Observational aspects, J. Atmos. Sci., 33 (1976), 1937-1954.

[4] P. J. Webster and J. Fasullo, Monsoon: Dynamical theory, in Encyclopedia of Atmospheric Sciences, Academic Press (2003), 153-168.

[5] E. Halley, An historical account of the trade winds, and monsoons, observable in the seas between and near the tropicks, with an attempt to assign the phisical cause of the said winds. Phil. Trans. Roy. Soc., 16 (1686), 1370-1385.

[6] S. Bordoni and T. Schneider, Monsoons as eddy mediated regime transitions of the tropical overturning circulation, Nature Geoscience, 1 (2008), 515-519. 
[7] T. Schneider and S. Bordoni, Eddy-mediated regime transitions in the seasonal cycle of a Hadley circulations and implications for monsoon dynamics, J. Atmos. Sci., 65 (2008), 915-934

\section{Long time impact of a small, slowly evolving, source term in a convection model \\ YANN BRENIER}

We consider the (generalized) convection mode 1

$$
\begin{gathered}
\partial_{t} v+(v \cdot \nabla) v-\alpha \Delta v+\nabla p=f, \quad \nabla \cdot v=0, \\
\partial_{t} f+(v \cdot \nabla) f=\varepsilon G(\varepsilon t, x, f(t, x))
\end{gathered}
$$

where $f=f(t, x)$ is advected by the velocity field $v$ with a small, slowly evolving, vector-valued, source term. We are interested in the impact of such a source term for large times of order $O\left(\epsilon^{-1}\right)$. After rescaling $(t, v(t, x))$ as $(t / \varepsilon, \varepsilon v(\varepsilon t, x))$, we get

$$
\begin{gathered}
\varepsilon^{2}\left(\partial_{t} v+(v \cdot \nabla) v\right)-\alpha \varepsilon \Delta v+\nabla p=f, \quad \nabla \cdot v=0, \\
\partial_{t} f+(v \cdot \nabla) f=G(t, x, f(t, x))
\end{gathered}
$$

We now consider the formal limit of these equations obtained by setting $\varepsilon=0$

$$
\begin{gathered}
\nabla p=y, \quad \nabla \cdot v=0, \\
\partial_{t} f+(v \cdot \nabla) f=G(t, x, f) .
\end{gathered}
$$

The main application is the case when $d=2$ and the source term

$$
G(x, y)=\left(x_{2}, y_{1}-x_{1}\right) .
$$

Then the limit equations coincide with the semigeostrophic equations in the special incompressible " $x-z$ " situation.

\section{Motivation For a CONVExity ASSUmption}

At first glance, the limit equations look strange since there is no evolution equation for $v$. However, $y$ is constrained to be a gradient. In three space dimension, for instance, since $v$ is divergence-free, we can write $v=\nabla \times A$ for some divergence free "potential vector" $A=A(t, x) \in R^{3}$. Then, by curling equation (6), we get a linear system for $A$, namely:

$$
\nabla \times\left(D_{x}^{2} p(t, x) \cdot \nabla \times A\right)=\nabla \times(G(x, \nabla p)),
$$

This system is elliptic in $A$ whenever

$$
c|\xi|^{2} \leq D_{x}^{2} p(t, x) \cdot(\xi, \xi) \leq c^{-1}|\xi|^{2}, \quad \forall t, x, \xi,
$$

for some constant $0<c<1$. This strong convexity assumption is therefore a natural solvability condition for the limit equations. 
However, this condition may break down in finite time. The simplest example is when

$$
G=-\nabla g(x)
$$

with $D^{2} g>0$. Indeed, then, a trivial global solution of both the original equations and their limit, is obtained just by setting:

$$
f(t, x)=\nabla p(t, x), \quad v(t, x)=0, \quad p(t, x)=p(0, x)-t g(x)
$$

which obviously violates condition (9) in finite time.

\section{MAin RESUlts}

Combining several recent results (quoted below), we can say:

i) the limit system admits local smooth solutions satisfying the strong convexity condition (9) and these solutions can be rigorously derived from the original equations as $\varepsilon \rightarrow 0$;

ii) existence of global (generalized) solutions $y=\nabla p \in C^{0}\left(R_{+} ; L^{2}\right)$ can be established under the weak convexity condition

$$
D_{x}^{2} p(t, x) \cdot(\xi, \xi) \geq 0, \quad \forall t, x, \xi .
$$

\section{REFERENCES}

[Br] Y. Brenier, Optimal transport, convection, magnetic relaxation and generalized Boussinesq equations. J. Nonlinear Sci. 19 (2009), no. 5, 547-570

[BC] Y. Brenier, M. Cullen, Rigorous derivation of the $x-z$ semigeostrophic equations. Commun. Math. Sci. 7 (2009), no. 3, 779-784.

[Lo] G. Loeper, A fully nonlinear version of the incompressible Euler equations: the semigeostrophic system, SIAM J. Math. Anal. 38 (2006) 795-823.

\section{The rapid decay of large-scale internal tides due to interactions with random small-scale topography OLIVER BÜHLER}

(joint work with Miranda Holmes-Cerfon)

The global-scale circulation of the ocean is intimately linked to unresolvable small-scale waves such as inertia-gravity waves, which owe their restoration mechanism to a combination of the stable stratification of the ocean (ie the fact that the water density increases with depth) and the Coriolis forces due to the Earth's rotation. Because these small-scale waves provide the lubrication for the functioning of the large-scale circulation of the ocean, there is a pressing need to improve our understanding of the dynamics of these waves, including their energy sources. An important energy source for these waves are the lunar tides, and an open problem in present-day oceanography is how this tidal wave energy is converted into the wave energy at the very small scales at which the lubrication can take place.

In this talk the interaction of tidal waves with small scale random undulations of the sea floor are investigated, which mathematically leads to an ill-posed problem 
of a type first studied by Sobolev. We solve this problem using a specialized numerical technique and show that the random topography is a very efficient wave energy converter and thus helps lubricating the ocean circulation.

\section{What sets the oceanic mid-depth stratification and overturning circulation? PaOla CESSI \\ (joint work with Christopher L. Wolfe)}

The processes maintaining the stratification in the oceanic mid-depth region (between approximately 1000 and $3000 \mathrm{~m}$ ) are explored using an eddy-resolving general circulation model in a simple geometry composed of a two-hemisphere, semi-enclosed basin with a zonally-reentrant channel in the southernmost eighth of the domain [1]. The mid-depth region lies below the wind-driven main thermocline but above the diffusively-driven abyssal ocean. Here, it is argued that the mid-depth stratification is determined primarily in the reentrant portion of the domain, which represents the Antarctic Circumpolar Current (ACC) region. Competition between mean and eddy overturning in the channel leads to steeper isotherms and thus deeper stratification throughout the basin than would exist without the channel. This is because in a reentrant geometry only mesoscale eddies can restratify the fluid by counteracting the wind-driven, thermally indirect cell. In the enclosed portion of the domain, basin-scale gyres can be supported which transport heat down-gradient, providing a much more effective restratification mechanism than mesoscale eddies. Thus in a geometry with an ACC region deep stratification is much stronger than in a fully enclosed geometry. Changes in wind forcing over the channel influence the stratification throughout the domain. Since the mid-depth stratification is controlled by adiabatic dynamics in the channel, it becomes independent of the interior diapycnal diffusivity, $\kappa$, as $\kappa \rightarrow 0$.

Isotherms which outcrop only in the channel are nearly horizontal in the semienclosed portion of the domain, whereas isotherms which also outcrop in the Northern Hemisphere $(\mathrm{NH})$ deviate from horizontal and are accompanied by geostrophically-balanced meridional transport. Along the set of isotherms that outcrop in both the ACC region and the NH, an adiabatic circulation can be established along the shared temperature surfaces, with diabatic processes confined to the surface mixed layer. In this configuration, a northern source of deep water can be supported, which leads to the formation of a thick mid-depth thermostad and a sizeable meridional overturning circulation (MOC) circulation which also shows a tendency to become independent of $\kappa$ as $\kappa \rightarrow 0$. A non-local scaling for the MOC is developed which relates the strength of the northern MOC to the depth of the isotherms at the equatorward edge of the southern channel. If there are no outcropping isotherms shared between the ACC region and the NH, the MOC depends on $\kappa$ and vanishes in the limit $\kappa \rightarrow 0$. 
The results of our simplified computations compare favorably to observations of large-scale neutral density in the world ocean.

\section{REFERENCES}

[1] Wolfe, C.L., and P. Cessi, What Sets the Strength of the Mid-Depth Stratification and Overturning Circulation in Eddying Ocean Models? , J. Phys. Oceanogr. 40 (2010), 15201538 .

\section{Statistics and interactions in deep cumulus convection George C. Craig}

Deep moist convection is a canonical example of a non-linear, non-Gaussian process in the atmosphere. The error growth timescale of order ten minutes is not resolved by the observing network, and is short in comparison to the timescales of interest for description and prediction of the atmospheric state. In effect, cumulus clouds influence the weather and climate as stochastic process - clouds appear out of nowhere - and can at best be described statistically. The distributions of clouds and precipitation in convective weather regimes are characterized by intermittency, associated with fat tails that deviate strongly from Gaussian. Indeed, active convective cores in a convecting region occupy only about $2-3 \%$ of the area, and a point-process model is likely to be more appropriate. The purpose of the line of research described briefly here is to use physical knowledge of cumulus convection to develop such a stochastic point process model, as an alternative to developing general approaches to non-linear, non-Gaussian systems that may be both complex and uneconomical. Cumulus clouds will be regarded as individual, well-separated entities, that interact through a small number of specific physical mechanisms. Since the main results described at the meeting are from published works, this abstract will simply direct the reader to the appropriate references.

Three interaction mechanisms between individual convective clouds can be identified. First, localized transfer of mass from lower to upper levels in the troposphere (effectively a dipole source-sink pair) generates horizontally propagating gravity waves that lead to a net subsidence and hence warming of the atmosphere between the clouds [1]. Temperature anomalies are thus communicated by wave propagation, leading to a characteristic response timescale for the convecting atmosphere as a whole that is given by the time taken for a gravity wave to cross the characteristic cloud spacing distance (typically a few tens of kilometers) [3]. A second interaction mechanism occurs when precipitation falls from the cloud into unsaturated air. The ensuing evaporation produces a pool of cold air at the ground that spreads as a density current and can initiate new convective clouds by lifting boundary layer air to its level of saturation. Since density currents travel slowly in comparison to deep gravity waves, and are eroded by surface heat fluxes, this effect is of short range (a few kilometers at most), and produces local clustering and in extreme cases an intense squall line. The third interaction mechanism is through the influence of lower tropospheric moisture on cumulus clouds, since 
buoyant updrafts are strongly inhibited by entrainment of dry air. Moisture can only be communicated from the cumulus clouds to their environment by advection, and since the clouds are inefficient at generated horizontal motions in the mid-troposphere, the timescale of this transport is set by the rate at which environmental air sinks from the upper troposphere where the clouds detrain, which is in turn set by the rate at which the air can give off heat through radiation, leading to a timescale of a month or more [9].

If the convective clouds are widely separated in space, it might be expected that they will feel the influence of the cloud field as a whole, rather than feeling a dominant effect of their nearest neighbors. In this limit, a simple mean field theory can be constructed [5], taking the form a spatial Poisson process, with a mark corresponding to the mass flux of the individual cloud. Comparison with explicit simulations of a field of convective clouds shows that the spatial distribution of convective mass flux can be predicted to within about $10 \%$, with the deviations taking the form of a clustering on scales of a few kilometers and a weak suppression over distances of 10-20 km, as might be anticipated from the first two interactions mechanisms above. The relatively good quantitative agreement of the mean field theory has motived its use as the basis for a stochastic cumulus parameterization for use in weather forecasting and climate models [7]. The third interaction mechanism (moisture advection) acts only weakly in the small domains used in the early simulations. There is a tendency for the domain to be divided into wet and dry regions, with all the convection occurring in the wet regions [8]. This effect is seen clearly in more recent simulations on larger domains [2], where over many weeks the convective clouds become concentrated in a single region, with the remaining atmosphere becoming very dry. It has recently been argued that the observed large-scale organization of convection has characteristics of a phase transition [6], a point of view that also follows naturally from the considerations listed here.

\section{REFERENCES}

[1] Bretherton, C. S. and P. K. Smolarkiewicz, Gravity waves, compensating subsidence and detrainment around cumulus clouds, J. Atmos. Sci. 46 (1989), 740-759.

[2] Bretherton, C. S., P. N. Blossey and M. Khairoutdinov, An energy-balance analysis of deep convective self-aggregation above uniform SST, J. Atmos. Sci. 62 (2005), 4273-4292.

[3] Cohen, B. G. and G. C. Craig, The response time of a convective cloud ensemble to a change in forcing, Quart. J. Roy. Meteor. Soc. 130 (2004), 933-944.

[4] Cohen, B. G. and G. C. Craig, Fluctuations in an equilibrium convective ensemble. Part II: Numerical experiments, J. Atmos. Sci. 63 (2006), 2005-2015.

[5] Craig, G. C. and B. G. Cohen, Fluctuations in an equilibrium convective ensemble. Part I: Theoretical basis, J. Atmos. Sci. 63 (2006), 1996-2004.

[6] Peters O. and J. D. Neelin, Critical phenomena in atmospheric precipitation, Nature Phys. 2 (2006), 393-396

[7] Plant, J. M., and G. C. Craig, A stochastic parameterization for deep convection based on equilibrium statistics, J. Atmos. Sci. 65 (2008), 87-105.

[8] Tompkins, A. M., and G. C. Craig, Radiative-convective equilibrium in a three dimensional cloud ensemble model, Quart. J. Roy. Meteor. Soc. 124 (1998), 2073-2097. 
[9] Tompkins, A. M., and G. C. Craig, Timescales of adjustment to radiative-convective equilibrium in the tropical atmosphere, Quart. J. Roy. Meteor. Soc. 124 (1998), 2693-2713.

\section{Subgrid scale closure for the Burgers equation based on stochastic mode reduction \\ Stamen IAnkov Dolaptchiev \\ (joint work with Ilya Timofeyev, Ulrich Achatz)}

Applying a systematic stochastic mode reduction strategy [1, 2] a local closure for the subgrid scale dynamics in the inviscid Burgers equation is constructed. Using an energy and momentum conserving finite difference discretization and introducing a fine and a coarse grid, the model variables are split into fast and slow modes. This is a different approach compared to previous studies, where the separation between the modes is done by truncation in EOF or Fourier space $[3,4]$. First, the closure assumptions for the stochastic mode reduction strategy are verified. Next, an effective stochastic model for the dynamics of the slow modes is presented. The model performs well in reproducing the variance and the autocorrelation function of the full model. The contributions of different terms in the subgrid scale model are discussed.

\section{REFERENCES}

[1] A. Majda, I. Timofeyev, E. Vanden-Eijnden, A mathematical framework for stochastic climate models, Commun. Pure Appl. Math. (2001), 0891-0974.

[2] A. Majda, I. Timofeyev, E. Vanden-Eijnden, A priori tests of a stochastic mode reduction strategy, Physica D 170 (2002), 206-252.

[3] C. Franzke, A. Majda, E. Vanden-Eijnden, Low-Order stochastic mode reduction for a realistic barotropic model climate, J. Atmos. Sci 62 (2005), 1722-1745.

[4] C. Franzke and A. Majda, Low-Order stochastic mode reduction for a prototype atmospheric GCM, J. Atmos. Sci 63 (2006), 457-479.

\section{Is potential temperature inversion complementary to potential vorticity inversion? \\ Joseph EGGER \\ (joint work with Klaus-Peter Hoinka, Thomas Spengler)}

Given the distribution of one atmospheric variable, that of nearly all others can be derived in balanced flow. In particular, potential vorticity inversion (PVI) selects $\mathrm{PV}$ to derive pressure, winds and potential temperature on the basis of a balance relation and for hydrostatic conditions. Potential temperature inversion (PTI) starts from available fields of PT to derive pressure, winds and PV. While PVI requires at least to invert a threedimensional elliptic operator with corresponding boundary conditions, PTI is easier to apply since one has to solve only an equation of first order, namely the hydrostatic equation with one boundary condition, in the simplest version. Nevertheless, PTI has hardly been used as a research tool 
in contrast to the popular PVI which has been applied widely also in the form of piecewise PVI.

The electrostatic analogy as discussed carefully by Bishop and Thorpe (1994) is a paradigm of PVI where it is claimed that local anomalies of PV exert an influence on the atmsophere just as do electric charges on the electric field. This view is exemplified by Bishop and Thorpe (1994) for a spherical PV anomaly. It is shown, however, that the quasigeostrophic solution to this problem as presented by Bishop and Thorpe (1994) contains potential temperature perturbations which cannot be induced by the spherical PV 'charge'. It follows that PV does not have any special inducing characteristics and is thus on equal terms with potential temperature which is conserved in adiabatic flow.

Idealized examples of PTI and PVI are compared. The inversions are equivalent but PTI is easier to perform and understand than PVI. ERA analyses are used to determine typical anomalies of PV and PT in the North Atlantic stormtrack region. Statistical form of PVI and PTI are applied to these anomalies. It turns out again that PTI is easier to understand than PVI.

\section{REFERENCES}

[1] Bishop, C., and A. Thorpe,1994: Potential vorticity and the electrostatic analogy: quasigeostrophic theory. Q. J. Roy. Met. Soc., 120, 713-731.

[2] Egger, J., and K.-P. Hoinka, 2010: Potential temperature and potential vorticity inversion: complemetary approaches. J. Atmos. Sci., 67, in print.

\section{Thermostats for point vortices}

$$
\text { JASON FRANK }
$$

(joint work with Svetlana Dubinkina, Ben Leimkuhler)

The statistical mechanics of a molecular dynamics system observed to be in thermal equilibrium with a heat reservoir at constant temperature is governed by the Gibbs distribution

$$
\rho(X)=Z^{-1} \exp -\beta H(X),
$$

where $X$ is the state of the system, $H(X)$ is the Hamiltonian, $\beta$ is the inverse statistical temperature, and $Z=\int \exp -\beta H(X) d X$ is the partition function. While it is straightforward to construct numerical integrators that conserve the Hamiltonian exactly, thereby allowing simulations at constant energy, the construction of an integrator that preserves the unique invariance - under the Liouville flow - of the measure (1), is significantly more challenging. Typically this is done by augmenting the vector field of the free dynamics with a thermostat device, such as was originally proposed by $[1,2]$ and later generalized by [3].

In [4] we introduce a generalized thermostat as a means of model reduction, with the motivation of applying this idea to point vortices and (in forthcoming work) other fluid models. The idea is to decompose a given system into resolved 
and unresolved variables, where the unresolved variables are treated as a thermal reservoir into and from which energy can be dumped or drawn. The energy exchange between the resolved variables and the reservoir is modeled with a thermostat. The resolved system is augmented with one or more additional degrees of freedom such that the dynamics on the extended phase space, when projected back onto the original phase space, preserves the desired measure.

Consider a dynamical system

$$
\dot{X}=f(X),
$$

whose vector field is assumed to be divergence-free $\operatorname{div} f \equiv 0$ and to possess a first integral $H(X)$. We introduce an auxiliary scalar variable $\zeta$, a perturbation vector field $g(X)$, and a function $h(X)$ such that phase flow of the augmented system

$$
\begin{aligned}
\dot{X} & =f(X)+\zeta g(X) \\
\dot{\zeta} & =h(X)-\zeta+\sqrt{\frac{2}{\alpha}} \dot{w}
\end{aligned}
$$

preserves the generalized product measure

$$
\tilde{\rho}(X, \zeta)=\hat{Z}^{-1} \exp -F(H(X)) \exp -\frac{\alpha}{2} \zeta^{2},
$$

where $F$ is a differentiable function, chosen to appropriately model the reservoir exchange (e.g. $F(H)=-\beta H)$. Under the above assumptions on $f$, the function $h(X)$ can be determined for any $g(X)$, leaving $\alpha$ and $g(X)$ as the only free parameters. It is important to choose $g$ to guarantee controllability if ergodic sampling is required. If this cannot be achieved with a single thermostat variable, the system can be augmented as above with additional thermostats. The rate of relaxation is controlled by $\alpha$.

The methodology was applied to a point vortex model originally proposed by Bühler in [5] to study the classical analysis of Onsager [6]. Bühler's system consisted of 4 strong 96 weak point vortices on a disc, where each set had an equal number of positive and negatively signed vortices. The statistics of the strong vortices were recorded under conditions corresponding to positive, negative and neutral inverse temperature regimes. In [4] we successfully reproduce Bühler's results using just the four strong vortices and the thermostat device. To obtain accurate results, it was necessary to model the finite reservoir effects of Bühler's direct simulations.

An important open question about this approach concerns the nonequilibrium behavior of the thermostated dynamics. Can one consider the dynamics to be predictive on short time scales? can correlations be computed accurately? In the context of gridpoint/PDE models additional open questions are how to model a reservoir of sub-grid scale energetics and the definition of appropriate perturbed vector fields.

\section{REFERENCES}

[1] S. Nosé, A molecular dynamics method for simulations in the canonical ensemble, Mol. Phys., 52 (1984), 255-268. 
[2] W.G. Hoover, Canonical dynamics: Equilibrium phase space distributions, Phys. Rev. A, 31 (1985), 1695-1697.

[3] A. Bulgac \& D. Kusnezov, Canonical ensemble averages from pseudomicrocanonical dynamics, Phys. Rev. A 42 (1990), 5045-5048.

[4] S. Dubinkina, J. Frank, B. Leimkuhler, Simplified modelling of energetic interactions with a thermal bath, with applications to a fluid vortex system, Multiscale Model. Simul., (2010), to appear.

[5] O. Bühler, Statistical mechanics of strong and weak vortices in a cylinder, Phys. Fluids, 14 (2002), 22139-2149.

[6] L. Onsager, Statistical hydrodynamics, Nuovo Cimento (9), 6 (1949), 279-287.

\section{Identification of climate switching during the last glacial using Bayesian statistical methods \\ Christian FranzKe \\ (joint work with Daniel Peavoy)}

Ice core data for the last glacial period indicate large temperature changes occurring on timescales of centuries or less. They are most pronounced in Greenland ice cores where they are characterised by a rapid warming of up to $10^{\circ} \mathrm{C}$, followed by a slow cooling period. They are known as Dansgaard-Oeschger (DO) events and were numbered and subjectively dated by eye [1]. There is evidence that the dramatic climate shifts at high latitudes in the Northern Hemisphere had global scale effects. For example, [2] observed an out of phase signal in Antarctic ice cores, whereas [3] note general agreement of temperature changes between one Greenland ice core (GISP2) and stalagmite records from the Hulu cave in China.

We propose the following model to systematically identify the DO events from ice core data. We assume that the time series can be modelled as a random walk dependent upon the state of the climate. This is intended to capture the differing distributions of the increments that occur during different climate regimes.

Under the random walk model the data $X_{i}$ at time $i$ evolves according to

$$
X_{i+1}=X_{i}+\mu_{S_{i}}+\sigma_{S_{i}} \epsilon_{i},
$$

where $\epsilon_{i} \sim \mathcal{N}(0,1)$ is the standard normal distribution, $\mu_{S_{i}}$ is the forcing and $\sigma_{S_{i}}$ is the standard deviation in state $S_{i}$. For $N$ data points, the probability of the data given the unobserved state sequence is

$$
P(X \mid \mu, \sigma, S)=\prod_{i=0}^{N-1} \mathcal{N}\left(X_{i+1}-X_{i}-\mu_{S_{i}}, \sigma_{S_{i}}^{2}\right) .
$$

To capture the uncertainty about the time spent in each climate regime an extra layer is introduced into the hierarchy that conditions $S$ on an unknown parameter $\lambda_{j}, j \in\{0 \ldots M\}$. This is the probability that $S_{i}$ is in state $j$, which is then distributed according to the multinomial distribution. In a Bayesian setting $\lambda_{j}$, and the other parameters, are random variables with a prior distribution. By defining conjugate priors the model can be inferred using a Gibbs sampling algorithm. The conjugate prior for $\lambda_{j}$ is given by a Dirichlet distribution with 
hyperparameter $\alpha$. This respects the constraint $\sum_{j} \lambda_{j}=1$. For $\sigma_{j}$ one defines $\tau_{j}=1 / \sigma_{j}^{2}$ and assigns a Gamma prior $\tau_{j} \sim \Gamma(a, b)$, whereas $\mu_{j}$ is a priori normally distributed $\mu_{j} \sim \mathcal{N}\left(0, \sigma_{\mu}^{2}\right)$. The hyperparameters $a, b$ and $\sigma_{\mu}$ are fixed. The posterior distribution can then be simulated by drawing sequentially from each of the conditionals. For $\mu_{j}$, we have

$\mu_{j} \sim \mathcal{N}\left(\frac{\left(\sum_{i=0}^{N-1} I\left[S_{i}=j\right] X_{i+1}-\Sigma_{i=0}^{N-1} I\left[S_{i}=j\right] X_{i}\right)}{\sum_{i=0}^{N-1} I\left[S_{i}=j\right]+\sigma_{j}^{2} / \sigma_{\mu}^{2}}, \frac{\sigma_{j}^{2} \sigma_{\mu}^{2}}{\sigma_{\mu}^{2} \sum_{i=0}^{N-1} I\left[S_{i}=j\right]+\sigma_{j}^{2}}\right)$,

where $I\left[S_{i}=j\right]=1$ if $S_{i}=j$ or 0 otherwise. The posterior for $\tau_{j}$ is the Gamma distribution

$$
\tau_{j} \sim \Gamma\left(a+\sum_{i=0}^{N-1} \frac{I\left[S_{i}=j\right]}{2}, b+\frac{1}{2} \sum_{i=0}^{N-1} I\left[S_{i}=j\right]\left(X_{i+1}-X_{i}-\mu_{S_{i}}\right)^{2}\right) .
$$

Each unobserved state is sampled from the updated multinomial distribution with probability

$$
P\left(S_{i}=j\right) \propto \lambda_{j} \sqrt{\tau_{j}} \exp \left(-\frac{\tau_{j}}{2}\left(X_{i+1}-X_{i}-\mu_{j}\right)^{2}\right) .
$$

By cycling through the above distributions one obtains a dependent sample from the posterior. As is usual for MCMC algorithms we discard an initial "burn in" period to allow for convergence to the posterior distribution.

Using this model we investigated the recurrence properties of the events. We found a significant signal at the 1450 year period for GISP2, agreeing with previous authors and supporting our method. This period is absent in the other data sets, in agreement with [4]. Due to the strong agreement between the climate regimes in two other Greenland ice cores (GRIP and NGRIP), and their recurrence times, we assign a higher significance to the results from these data sets. We conclude that there is no lasting periodic signal for the rapid warming or cooling events of the last glacial period and suppose that the apparent periodicity seen in GISP2 is due to a small number of events that occur at the regular interval of 1450-1500 years.

More background and details of this study can be found in [5].

\section{REFERENCES}

[1] Dansgaard, W., Johnsen, S. J., Clausen, H. B., Dahl-Jensen, D., Gundestrup, N. S., Hammer, C. U., Hvidberg, C. S., Steffensen, J. P., Sveinbjornsdottir, A. E., Jouzel, J. and Bond, G.: Evidence for general instability of past climate from a 250-kyr ice-core record, Nature, 364, (1993), 218-220.

[2] Blunier, T., Chappelliaz, J., Schwander, J., Dallenbach, Stauffer, B., Stocker, T. F., Raynaud, D., Jouzel, J., Clausen, H. B., Hammer, C. U. and Johnsen, S.J.: Asynchrony of Antarctic and Greenland climate change during the last glacial period. Nature, 384, (1998), 739-743.

[3] Wang, Y. J., Cheng, H., Edwards, R. L., An, Z. S., Wu, J. Y., Shen, C.-C. and Dorale, J. A.: A High-Resolution Absolute-Dated Late Pleistocene Monsoon Record from Hulu Cave, China, Science, 294, (2001), 2345-2348. 
[4] Ditlevsen, P. D., Kristensen, M. S. and Andersen, K. K.: The Recurrence Time of Dansgaard - Oeschger Events and Limits on the Possible Periodic Component, J. Climate, 18, (2005), 2594-2603.

[5] Peavoy, D. and C. Franzke: Objective identification of climate states from Greenland ice cores for the last glacial period, Climate of the Past Discussions, 6, (2010), doi:10.5194/cpd6-1209-2010.

\section{Energy transports and tropical precipitation shifts in simulations of 20th and 21st century climate \\ DARGAN M. W. Frierson \\ (joint work with Yen-Ting Hwang, Sarah M. Kang)}

Most state-of-the-art global climate models (GCMs) predict an increase in atmospheric poleward energy transport with global warming; however, the amount of increase varies significantly from model to model. The differences among models are interpreted with a one-dimensional partial differential equation (PDE) model that diffuses moist static energy, similar to that found in [1]. By prescribing radiative forcings and feedbacks, and changes in ocean circulation and ocean heat uptake from the GCMs, the PDE model with constant diffusivity is able to explain the spread among models. The increase in poleward transport in all models is due to increases in moisture content of the atmosphere. This work suggests that radiative feedbacks and forcings dominate dynamical changes in eddies to determine atmospheric energy transport changes, and that biases in feedbacks and forcings will not only affect climate locally but will also influence other latitudes through energy transports.

Even more different among GCM simulations are the predictions of zonally averaged tropical precipitation. Some models predict increases of several tens of centimeters per year at the same latitude where other models predict decreases in precipitation. Much tropical precipitation on Earth happens in relatively narrow bands such as the inter-tropical convergence zone (ITCZ), so the question of whether the ITCZ shifts northward or southward with warming is key to the future of tropical precipitation. We show that the one-dimensional PDE model described above can also explain the behavior of the ITCZ in the models. Models with more positive radiative feedbacks/forcings in the Northern Hemisphere have more of a northward shift, with terms closer to the equator being especially effective in shifting the ITCZ. The cross-equatorial energy transport predicted by the simple model is well-correlated with the ITCZ shifts in the GCMs, as described in the theory of [2].

The simple model also allows attribution of the ITCZ shifts. We find that aerosol cooling of the Northern Hemisphere in the 20th Century is key to the modeled southward shifts of the ITCZ, with cloud feedbacks causing a significant spread among models. 


\section{REFERENCES}

[1] Frierson, D. M. W., Held, I. M. and P. Zurita-Gotor, A gray-radiation aquaplanet moist GCM. Part II: Energy transports in altered climates, J. Atmos. Sci., 64 (2007), 1680-1693, doi:10.1175/JAS3913.1.

[2] Kang, S. M., Held, I. M., Frierson, D. M. W. and M. Zhao, The response of the ITCZ to extratropical forcing: Idealized slab-ocean experiments with a GCM, J. Climate, 21 (2008), 3521-3532, doi:10.1175/2007JCLI2146.1.

\section{On the time scales of midlatitude atmospheric variability}

\section{EDWIN P. GERBER}

The processes that set the time scales of internal variability in the midlatitude atmosphere are investigated in a hierarchy of systems, from the barotropic vorticity equation on the sphere to coupled atmosphere-ocean climate prediction models. The dominant patterns of variability in the extratropics are the annular modes, which characterize meridional shifts of the midlatitude westerly jets [5]. Even a relatively modest shift in the midlatitude jets can make a big difference on a local level. In the northeastern United States, for example, a jet shift of a few degrees just a couple grid boxes in a typical General Circulation Model - is the difference between record snow in Washington, DC and a typical wintry weekend for Boston. The persistence of these shifts on intraseasonal time scales makes the difference between a lone storm and a very cold winter.

The persistence of the midlatitude jet shifts is characterized by the e-folding time scale of the annular mode. This time scale varies as a function of height and season, exhibiting a curious asymmetry between the two hemispheres: it peaks during the boreal winter in the Northern Hemisphere and the austral fall/early summer in the Southern Hemisphere [1]. Comprehensive models capture many of the key features of the seasonal cycle in annular mode time scales, including the interhemispheric asymmetry, but exhibit significant biases, particularly in the Southern Hemisphere summer where the time scales are too long [2]. A model's ability to capture the variability appears related to its ability to capture the time mean climatology. Models with an equatorward bias of the midlatitude jet exhibit longer annular mode time scales [4]. Systems of intermediate complexity help illustrate the connection between jet position and the annular mode time scale, and explain why model biases tend to be largest in the Southern Hemisphere. In particular, the contract in behavior between a barotropic [6] and baroclinic [3] model of the atmosphere highlights the importance of interactions between baroclinic eddies and the zonal mean flow in setting the time scale of the annular modes.

\section{REFERENCES}

[1] Baldwin, M. P., D. B. Stephenson, D. W. J. Thompson, T. J. Dunkerton, A. J. Charlton and A. O'Neill, Stratospheric Memory and Skill of Extended-Range Weather Forecasts, Science, 301 (2003), 646-640. 
[2] Gerber, E. P., L. M. Polvani, and D. Ancukiewicz, Annular mode time scales in the Integovernmental Panel on Climate Change Fourth Assessment Report models, Geophys. Res. Lett., 35 (2008), doi:10.1029/2008GL035712.

[3] Gerber, E. P. and G. K. Vallis, Eddy-zonal flow interactions and the persistence of the Zonal Index, J. Atmos. Sci., 64 (2007), 3296-3311.

[4] Kidston, J. and E. P. Gerber, Intermodel variability of the poleward shift of the austral jet stream in the CMIP3 integrations linked to biases in $20^{\text {th }}$ century climatology, Geophys. Res. Lett., 37, doi:10.1029/2010GL042873.

[5] Thompson, D. W. J. and J. M. Wallace, Annular modes in the extratropical circulation. Part I: Month-to-month variability, J. Climate, 13 (2000), 1000-1016.

[6] Vallis, G. K., E. P. Gerber, P. J. Kushner, and B. A. Cash, A mechanism and simple dynamical model of the North Atlantic Oscillation and annular modes, J. Atmos. Sci., 61 (2004), 264-280.

\section{Identifying and predicting the extensional and meandering phases of the jet in a double-gyre ocean model \\ Dimitrios GiannAKIS \\ (joint work with Andrew J. Majda)}

Large-scale oceanic circulation plays an important role in climate variability on interannual, interdecadal, and longer timescales. Intensified eastward currents (e.g., the Gulf Stream and the Kuroshio current), which are ubiquitous features of the Earth's ocean basins, exhibit a pronounced metastable behavior, characterized by transitions between persistent regimes, with lifetimes spanning several months to several years. The origin of regime behavior on these timescales (which are of high relevance to human activity and mid-term climate change) is generally attributed to the nonlinear response of the ocean to steady, large-scale wind-stress forcing, rather than systematic changes in solar forcing and/or thermohaline effects.

The essential physics of wind-driven ocean circulation are adequately described by reduced-barotropic quasigeostrophic (QG) models [6]. Driven by a doublegyre forcing (mimicking the subpolar and subtropical gyres in ocean basins), these models develop an eastward, Gulf-Stream-like jet separating from the western domain boundary. Here, the empirical phenomenology is that regime behavior is organized around three states, characterized by low, middle, and high kinetic and potential energy. The main feature of the high-energy (or extensional) phase is the large penetration length and small curvature of the jet. In the low-energy state, the jet is significantly weaker, and follows a meandering path. The middle-energy state spatially resembles the steady-state temporal average of the flow field.

In this talk we discuss a method based on finite-element (FEM) data clustering $[3]$ and empirical information theory $[4,5]$ for identifying and predicting the circulation regimes in this class of ocean models. Our data-driven approach involves using numerical realizations of the full model (in this case, the 1.5-layer model by McCalpin \& Haidvogel [6]) to build low-order dynamical models that associate each circulation regime with a cluster in the phase space of the full model, and describe the transitions between the regimes as a Markov jump process [7]. 
Central to the procedure is the notion of temporal coarse graining, implemented here via FEM discretization of the input time series for data clustering. We describe how coarse graining induces the physically appealing property of timeregularity of cluster affiliations, and demonstrate that it results to superior quality of phase-space partitioning and long-term (decadal) prediction skill compared to standard $K$-means clustering. Throughout, we assess the quality of phase-space partitioning and predictive skill by means of the relative entropy between timedependent probabilities for the total energy conditioned on the clusters and the climatological distribution [1]. We also employ relative entropy to estimate a posteriori the error in modeling the transitions between the states as a Markov jump process.

Our main results [2] are that (i) the optimal partitioning of the phase space of the full model is into three clusters, characterized by low, middle and high expected values of the total energy; (ii) the spatial configuration of the streamfunction associated with these clusters is in excellent agreement with the meandering, steady-state, and extensional phases of the jet; and (iii) decade-horizon predictions about the regime of the model can be made by coarse observations spanning $2-3$ years. Thus, our results provide independent and objective verification of the empirical phenomenology of the 1.5-layer model. Our method for identifying circulation-regimes and making long-term forecasts should be applicable to more comprehensive ocean models.

\section{REFERENCES}

[1] D. Giannakis \& A. J. Majda, Entropy-based measures for model error and predictive skill of coarse-grained climate models (2010), in preparation.

[2] D. Giannakis \& A. J. Majda, Identifying and predicting the extensional and meandering phases of the eastward jet in a simple double-gyre ocean model (2010), in preparation.

[3] I. Horenko, On the identification of nonstationary factor models and their application to atmospheric data analysis, J. Atmos. Sci., 67(5) (2010), 1559.

[4] A. J. Majda, R. V. Abramov \& M. J. Grote, Information Theory and Stochastics for Multiscale Nonlinear Systems, CRM Monograph Series, 25, American Mathematical Society (2005).

[5] A. J. Majda \& B. Gershgorin, Quantifying uncertainty in climate change science through empirical information theory, Proc. Natl. Acad. Sci., 32 (2010), in press.

[6] J. D. McCalpin \& D. B. Haidvogel, Phenomenology of the Low-Frequency Variability in a Reduced-Gravity Quasigeostrophic Double-Gyre Model, J. Phys. Oceanogr., 26 (1996), 739.

[7] P. Metzner, E. Dittmer, T. Jahnke \& C. Sch utte, Generator estimator of Markov jump processes based on incomplete observations equidistant in time, J. Comput. Phys., 227(1) (2007), 353. 
Development of the non-hydrostatic unified model of the atmosphere (NUMA)

Francis X. Giraldo

(joint work with James Kelly, Emil Constantinescu)

The current vision at operational numerical weather prediction centers is to develop unified (global to mesoscale) models that can be used for global NWP as well as for regional modeling. We have seen this trend already in Canada (with GEM) and the UK (with the Unified Model) but are also seeing it in Japan (with NICAM) and Germany (with ICON), although these last two systems are primarily focused on the climate problem. In the U.S., there is currently a joint effort between NOAA (National Oceanic and Atmospheric Admininstration) and the Navy to develop a unified model for both NWP (5-7 day forecasts) and for decadal simulations (10-30 years). The unifying theme in all of these efforts is "multi-scale" modeling, meaning that both operational NWP and climate research centers are focusing on developing a model that is applicable across a disparate range of scales; the reason for this is economics (the reduced cost in maintaining only one model per organization). From a science perspective, this opens the door for the construction of radically new models that must use fewer underlying physical simplifications (e.g., cannot use the hydrostatic approximation). In addition, computing power has reached a sufficiently high-level that it is now possible to propose the use of the anelastic, non-hydrostatic, or pseudo-incompressible equations instead of the hydrostatic equations that have been typically used for global models (such as in NWP and in climate GCMs).

In this talk, I will describe our strategy for constructing such unified models. It turns out that addressing this issue from the standpoint of a modeling framework simplifies the model construction significantly. What I mean by a modeling framework is that the model is constructed by increasing levels of complexity that are all built within the same infrastructure. This simplifies the validation by adding new components to the existing ones in a hierarchical approach. For example, one could first begin by constructing a model that simulates the transport of a scalar variable; this will tell you if you are doing the transport properly. Then one adds a few more variables to solve the shallow water equations, until one gets to the compressible Navier-Stokes equations. Finally, one can add the proper vectors to be able to handle not just flow in a box (as in mesoscale applications) but also flow on a sphere (as in global applications).

\section{Estimation of the sensitivity of atmospheric systems using fluctuation-dissipation theorem and unstable periodic orbits ANDREY GRITSUN}

In this study we discuss two possibilities to estimate response operators of the statistical characteristics of (chaotic, dissipative) atmospheric system onto small external forcing. 
The first method is based on applying fluctuation-dissipation theorem (FDT) which states ([1],[2]) that for systems with stationary quasi-Gaussian PDF the response of the system statistical characteristics to weak external forcing could be expressed in terms of covariances and lag-covariances of fluctuations of the undisturbed system. C.Leith ([3]) expressed an idea that the dynamics of the earth's atmosphere is reasonably close to the conditions required by FDT and suggested to use FDT in climate studies. The major advantage of this approach is that one may calculate climate system approximate response operator (relating changes in the system statistics with changes in the system external forcing) using observational data only.

In this study we use FDT to construct approximate response operators for the atmospheric general circulation model CCM0 of National Center for atmospheric research. It is demonstrated that with these operators one may solve inverse problem of finding external forcing producing a prescribed response of the system average state with sufficient accuracy (see also [4], [5]). Another important application is the analysis of the system sensitivity (i.e. the calculation of the external forcing that produces the maximum possible response of the system).

Second approach for calculation of the system response operator onto small forcing is based on the approximation of the system invariant measure using unstable periodic orbits (UPOs) of the system. Indeed, UPOs are dense on the system attractor for axiom A system and any trajectory of this system can be approximated by some orbit with any given accuracy $([6],[7])$. The system invariant measure could also be expressed in terms of UPOs characteristics. Under this approximation orbits are taken into account according to their weights related to their instability properties ([8]). As a rule, models describing the dynamics of the atmosphere are not axiom A systems but belong to a wider class of systems with nonzero Lyapunov exponents. Nevertheless, there are grounds to suppose that such systems have a sufficient number of unstable periodic solutions forming the skeleton of the system attractor ([9]).

In this study we are using above approach to estimate the response operator of a simple barotropic atmosphere model to small external perturbation consisting in a change of the external forcing of the system. Approximately 2000 unstable periodic trajectories were found for the system ([10]). Orbits approximate the PDF of the system and its statistical characteristics with reasonable accuracy ([11]). Response operators of the system mean state to small external perturbations were calculated for different settings of UPO weight coefficients. It is shown that under a correct choice of the weight function one can reproduce the response operator with a good accuracy. Finally, we analyze the relationships between UPOs and system modes of variability $([12])$.

\section{REFERENCES}

[1] U. Deker, F. Haake Fluctuation-dissipation theorems for classical processes, Phys. Rev. A11 (1975), 2043

[2] H. Risken The Fokker-Plank Equation: Methods of Solution and Applications, SpringerVerlag, New York, 1984, 454pp. 
[3] C.E. Leith Climate response and fluctuation dissipation, J. Atmos. Sci., 32 (1975), 20222026.

[4] A. Gritsun, Branstator G., Majda A. Climate response of linear and quadratic functionals using the fluctuation-dissipation theorem, J. Atmos. Sci., 65 (2008), 2824-2841.

[5] A. Gritsun, Branstator G. Climate response using a three-dimensional operator based on the fluctuation dissipation theorem, J. Atmos. Sci., 64 (2007), 2558-2575.

[6] D. Auerbach D., P. Cvitanovic, J.-P. Eckmann, G. Gunaratne, I. Procaccia Exploring chaotic motion through periodic orbits, Phys. Rev. Lett., 58 (1987), 2387-2389.

[7] R. Bowen Periodic points and measures for axiom A diffeomorphisms, Trans. Amer. Math. Soc., 154 (1971), 377-397.

[8] D. Ruelle Smooth dynamics and new theoretical ideas in nonequilibrium statistical mechanics, J. Statist. Phys, 95 (1999), 393-468.

[9] G. Gallavotti Chaotic dynamics, fluctuations, nonequilibrium ensembles, Chaos, 8 (1998), N2, 384-392.

[10] A. Gritsun Unstable periodic trajectories of a barotropic model of the atmosphere, Russ. J. Numer. Anal. Math. Modelling, 23 (2008), N4, 345-367.

[11] A. Gritsun Unstable periodic orbits and sensitivity of the barotropic model of the atmosphere, Russ. J. Numer. Anal. Math. Modelling, 25 (2010), N4, 303-321.

[12] A. Gritsun Statistical characteristics of of barotropic atmospheric system and its unstable periodic silutions, Doklady Earth Sciences, (2010), accepted.

\section{Entropy, targeted, and redundant observations for filtering turbulent} signals

\section{Marcus J. Grote}

(joint work with Andrew J. Majda)

Many contemporary problems in science involve making predictions based on partial observation of extremely complicated spatially extended systems with many degrees of freedom and with physical instabilities on both large and small scale. Various new ensemble Kalman filtering strategies have been developed for these applications and new mathematical issues arise. Recently, Majda et al. developed explicit off-line test criteria for stable accurate discrete filtering [1, 2] and various reduced Fourier domain Kalman filters for spatially extended turbulent systems [3]. Here we extend the analysis and explore the filter skill to the situation of sparse irregular (non-equispaced) observations, in particular for the prediction of large-scale singular events. By using the relative entropy and the Shannon entropy difference to measure the information content we develop new strategies for removing redundant observations [4] and positioning optimal targeted observations.

\section{REFERENCES}

[1] M.J. Grote and A.J. Majda, Stable Time Filtering of Strongly Unstable Spatially Extended Systems, Proc. Natl. Acad. Sciences 103 (2006), 7548-7553.

[2] A.J. Majda and M.J. Grote, Explicit Off-line Test Criteria for Stable Accurate Time Filtering of Strongly Unstable Spatially Extended Systems, Proc. Natl. Acad. Sciences 104 (2007), $1124-1129$.

[3] J. Harlim, A. J. Majda, Mathematical Strategies for Filtering Complex Systems: Regularly Spaced Sparse Observations, J. of Comp. Phys. 227 (2008), 5304-5341. 
[4] Q. Xu, Measuring information content from observations for data assimilation: relative entropy versus shannon entropy difference, Tellus A 59 (2007), 198-209.

\section{Nonstationarity in discrete multifactor models, memory and application to cloud modeling \\ ILLIA HORENKO}

Unified framework for identification of models with discrete state space (discrete independent variable models and discrete Markov chains) is introduced [1]. It extends the recently developed methods of nonstationary Markov model parameterisation [2] towards indirect ensemble observations under influence of multiple exogenous factors and allows to test whether the available observational or simulation data is best described via the stationary or nonstationary, Markovian or independent processes. It also allows estimation of the relative significance of the exogenous factors on the process dynamics. The resulting numerical algorithm is applied to analysis of the total relative cloud cover data in Europe, interpreted as a discrete probability density of an ensemble of discrete microscopic cloud models switching between the two states: cloud and no-cloud states. The obtained discrete data-based cloud models are analyzed wrt. their memory, stationarity and relative importance of external factors (such as surface air temperature, total column water quantity, North-Atlantic and Arctic oscillations indices). The quality of online predictions based on the new method is compared with the results obtained by standard linear and stationary methods of time series analysis.

\section{REFERENCES}

[1] I. Horenko, Nonstationarity in discrete multifactor models, memory and application to cloud modeling, submitted to J. of Atmos. Sci. (2010).

[2] I. Horenko, On Robust Estimation of Low-Frequency Variability Trends in Discrete Markovian Sequences of Atmospheric Circulation Patterns, J. of Atmos. Sci. 66 (2009), 2059-2072.

\section{Simulation of the MJO in a coarse resolution GCM using a simple multicloud parametrization BOUALEM KHOUIDER \\ (joint work with Andrew Majda, Amik St-Cyr, Joseph Tribbia)}

The Madden Julian oscillation (MJO) is the dominant feature of the low frequency variability of the tropical atmosphere. It is observed to propagate over the Indian Ocean/Western Pacific warm pool region at a slow speed of about $5 \mathrm{~m} / \mathrm{s}$ with a period of 40 days and a wavelength of about $20,000 \mathrm{~km}$ in the form of an envelope of higher frequency organized cloud clusters and super-clusters (e.g. [9]). The $\mathrm{MJO}$ is believed to affect strongly the tropical and extra-tropical climate and weather patterns such as monsoons, ENSO, tropical cyclones, and teleconnection patterns, e.g. the North Atlantic and Pacific North American oscillations [1], which modulate the weather in the Northern Hemisphere midlatitudes. However, 
current operational general circulation models (GCMs) used for climate and longrange weather predictions represent poorly the MJO and organized convection on synoptic scales known as convectively coupled waves (CCW) and thus fail to capture accurately the large-scale circulation associated with organized convection in the tropics. Although poorly understood, this poor performance of GCMs is often associated with the inadequate treatment of organized convection by the underlying cumulus parametrizations.

While the synoptic scale CCWs are recognized as being the moist equivalents of the equatorially trapped normal modes, the spectral peak associated with the MJO outgoing longwave radiation (a proxy for convective precipitation) appears completely separated from the dispersion relation curves of the equatorial waves, in the frequency-wavenumber domain [8]. Detailed observations reveal the persistence of three cloud types (congestus, deep and stratiform) that characterize organized convective systems in the tropics on a long range of scales, from the individual convective cell of about 1 to $10 \mathrm{~km}$ up to the planetary scale MJO [2]. During the recent years K. and Majda introduced and analyzed a simple mathematical model based on the three cloud types that characterize organized tropical convection with plausible interactions of the clouds with each other and with the large scale flow. The so-called multicloud model is very successful in reproducing very realistic convectively coupled waves both in linear theory and in non-linear simulations based on a simple model with crude vertical resolution reduced to the first two baroclinic modes of vertical structure [3, 5, 6, etc]. In this talk we present numerical simulation results of two benchmark examples for the MJO and CCWs, in realistic parameter regimes characterized by high and low moisture content in the lower troposphere, respectively, using the multicloud model as a cumulus parametrization in the next generation of the U.S. National Centre for Atmospheric Research GCM (HOMME: High Order Methods Modelling Environment) at low resolution on the order of $170 \mathrm{~km}$ grid mesh in the horizontal and 26 vertical levels. The so-coupled model demonstrated very high skill in reproducing realistic MJO-like and synoptic scale CC waves with physical and morphological features resembling the observational record. The details of the model coupling and discussion of the results are reported in [7].

\section{REFERENCES}

[1] N. Bond and G. Vecchi, The influence of the Madden-Julian Oscillation on precipitation in Oregon and Washington. Wea. Forecasting 18 (2003), 600-613.

[2] R. H. Johnson, R. H., T. M. Rickenbach, S. A. Rutledge, P. E. Ciesielski, and W. H. Schubert, Trimodal characteristics of tropical convection. J. Climate 12 (1999), 2397-2407.

[3] B. Khouider and A. J. Majda, A simple multicloud parametrization for convectively coupled tropical waves. Part I: Linear analysis. J. Atmos. Sci. 63 (2006), 1308-1323

[4] B. Khouider and A. J. Majda, A simple multicloud parametrization for convectively coupled tropical waves. Part I: nonlinear simulations. J. Atmos. Sci. 64 (2007), 381-400.

[5] B. Khouider and A. J. Majda, Multicloud models for organized tropical convection: Enhanced congestus heating. J. Atmos. Sci. 65 (2008), 895-914.

[6] B. Khouider and A. J. Majda, Equatorial convectively coupled waves in a simple multicloud model. J. Atmos. Sci. 65 (2008), 3376-3397. 
[7] B. Khouider, A. St-Cyr, A. J. Majda, and J. Tribbia MJO and convectively coupled waves in a coarse resolution GCM using a simple multicloud model. J. Atmos. Sci. (Submitted).

[8] M. Wheeler and G. N. Kiladis, Convectively coupled equatorial waves: Analysis of clouds and temperature in the wavenumber-frequency domain. J. Atmos. Sci. 56 (1999), 374-399.

[9] C. Zhang, Madden-Julian oscillation. Rev. Geophys. 43 (2005), RG2003, doi:10.1029/2004RG000158.

\section{On A Mimetic Discretization of the Ocean Primitive Equations PETER KorN}

Ocean Primitive Equations. Current state-of-the-art numerical ocean climate models are based on the incompressible Navier-Stokes equations on a rotating sphere with a free surface, subject to the hydrostatic and Boussinesq approximation. The equations are completed by advection-diffusion equations for salinity and temperature and an equation-of-state that relates density to salinity and temperature. The resulting set of equations is called the "ocean primitive equations"' (PE), (for details see [1]).

Velocity : $\quad \partial_{t} \mathbf{v}+(f+\omega)_{k} \times \mathbf{v}+w \partial_{z} \mathbf{v}+\frac{\nabla|\mathbf{v}|^{2}}{2}+\frac{\nabla p}{\rho_{0}}-\nabla \cdot\left(\mathcal{K}_{v} \nabla \mathbf{v}\right)=\mathcal{F}_{\mathbf{v}}$,

Hydrostatic : $\quad \partial_{z} p=-\rho g$,

Incompress. : $\quad \nabla \cdot \mathbf{v}=0$

Free Surface : $\quad \partial_{t} h+\nabla \cdot(h \mathbf{v})=\mathcal{F}_{h}$,

Tracer : $\quad \partial_{t} C+\nabla \cdot(C \mathbf{v})-\nabla \cdot\left(\mathcal{K}_{C} \nabla C\right)=\mathcal{F}_{C}$,

Eq. of State: $\quad \rho=\rho(C)$.

We describe a new discretization of these equations that is based on the method of"mimetic finite differences" (MFD).

Mimetic Discretization Method. The idea of MFD is to discretize continuous equations by defining (discrete) difference operators that mimic the behavior of (continuous) differential operators and implement within the discrete setting relations from vector calculus such as the Theorems of Gauss and Stokes. This is fundamentally different from classical finite differences that rely on Taylor expansions. MFD, pioneered by J. Hyman, M. Shashkov and F. Brezzi, can be described by the following four generic steps

(1) Specify the degrees of freedom of the discrete variables and their location, i.e. choose discrete spaces for velocity, pressure etc.

(2) Specify scalar product for the discrete velocity and pressure spaces.

(3) Choose a "primal operator" (e.g. divergence) and discretize by vector calculus.

(4) Define the "dual operator" (gradient) via primal operator and the scalar product.

The grid that we use consists of a Delaunay triangulation of the sphere with a associated Voronoi grid of hexagons. On this grid we use a so-called C-grid staggering: scalar variables such as pressure are assumed to be constant within 
a triangle and are located at the triangles circumcenter, the normal component of the velocity vector is constant along an edge and resides at the midpoint of a triangle edge and the vorticity, which is constant on a dual hexagon, sits at the vertices of triangles. In the vertical direction we use a z-ccordinate system.

The Discretized Primitive Equations. The variable staggering requires a consistent (linear) mapping from cell centers to edges and from vertices to edges and back. In order to avoid ad-hoc interpolations we start from a discrete weak form of the PE. An important observation is that the weak form requires that the mapping $Q$ from cell centers to edges has to be the transposed of the mapping $P$ from edges to cells, i.e. $Q=P^{T}$. The composite $P^{T} \circ P$ then defines an inner product on the discrete velocity space. Finally this leads to the discrete ocean PE:

$$
\begin{array}{cc}
\text { Velocity : } & \frac{d}{d t} P^{T} P v_{e, k}+\hat{P}^{T}((\omega+f) \hat{P} v)_{e, k}+P^{T}\left({\overline{w_{c, k+\frac{1}{2}} \partial_{z} P v_{k}}}^{z}\right) \\
& +P^{T} P \nabla\left(\frac{|P v|_{k}^{2}}{2}+\frac{p_{k}}{\rho_{0}}\right)-P^{T} \nabla \cdot \mathcal{K}_{v} \nabla P v_{e, k}-\partial_{z} A_{v} \partial_{z} P^{T} P v_{e, k}=\mathcal{F}_{v}, \\
\text { Hydrostatic : } & \partial_{z} p=-\bar{\rho} g, \\
\text { Incompress. : } & \nabla \cdot\left(P^{T} P v\right)+\partial_{z} w=0, \\
\text { Freesurface : } & \frac{\partial h}{\partial t}+\nabla \cdot\left[P^{T}\left(\int_{-H}^{h} P v d z\right)\right]=\mathcal{F}_{h}, \\
\text { Tracer : } & \frac{d C_{c, k}}{d t}+\nabla \cdot\left[P^{T}(C P v)\right]_{c, k}-\nabla \cdot \mathcal{K}_{C} \nabla C_{c, k}-\partial_{z} A_{C} \partial_{z} C_{c, k}=\mathcal{F}_{C},
\end{array}
$$

$k$ denotes a vertical level and ${ }^{-z}$ a vertical interpolation. Examples from [2] for the edge-to-cell mapping are $\overrightarrow{P v}_{K}:=\frac{1}{|K| \Delta z_{K}} \sum_{e} v_{e}|e| \triangle z_{e}\left(\vec{x}_{e}-\vec{x}_{K}\right)$, with $\vec{x}_{K}$ the position of the center of triangle $K, \vec{x}_{e}$ the edge midpoint, $|e|$ the edge length and $\triangle z_{e}$ height at edge $e$. The tranposed operator is $\left.P^{T} F\right|_{e}:=\frac{1}{\left|\vec{x}_{K}-\vec{x}_{L}\right|}\left\{\vec{F}_{K} \cdot\left(\vec{x}_{e}-\right.\right.$ $\left.\left.\vec{x}_{K}\right)-\vec{F}_{L} \cdot\left(\vec{x}_{e}-\vec{x}_{L}\right)\right\}$, with $e$ the edge between triangles $K$ and $L$. The mapping $\hat{P}, \hat{P}^{T}$ between edges and vertices has an analogous form on the dual Voronoi grid.

Properties of Discrete Equations. It can be shown that the discretized PE conserve mass and total energy. If one applies the two-dimensional equivalent of the discretization above to the shallow-water equations, then enstrophy is additionally conserved. The Nullspace of the discretized Coriolis operator contains the Null-vector only, in accordance with the continuous Coriolis operator. This avoids a common problem of many C-grid discretizations that have a large Null-space, a fact that may create artificial modes. For the specific pair of mappings $P, P^{T}$ and $\hat{P}^{T} \hat{P}$ from [2] all properties translate from a triangular C-grid to a $\mathrm{C}$-grid staggering on arbitrary polygons. Many of the described discretization properties do not depend on the specific functional form of the edge-to-cell and cell-to-edge mapping but on their transposed character. In this sense we have suggested a whole family of PE discretizations, parametrized by the metric $P^{T} \circ P$.

\section{REFERENCES}

[1] S. Griffies, Climate Ocean Modelling, Princeton University Press, (2004).

[2] B. Perot, Conservation Properties of Unstructured Staggered Mesh Schemes, J. Comp. Phys. 159, (2000), 58-89 
[3] P. Korn, An Enstrophy and Energy Conserving Scheme for the Shallow Water Equations on a $C$-grid, (in preparation)

[4] P. Korn, A Mimetic Discretization of the Ocean Primitive Equations, (in preparation)

\section{Stochastic subgrid-scale parametrisation using cluster-weighted modelling \\ FRANK KWASNIOK}

The dynamics of weather and climate encompass a wide range of spatial and temporal scales which are coupled through the nonlinear nature of the governing equations of motion. This gives rise to the closure problem; any weather or climate model needs a parametrisation scheme to account for the impact of unresolved scales and processes. A stochastic climate model resolves only a limited number of large-scale, low-frequency modes; the effect of unresolved scales and processes onto the resolved modes is represented by stochastic terms. Here, such low-order stochastic models are derived empirically from time series of the system using statistical parameter estimation techniques.

Firstly, we explore the proposed stochastic closure scheme using the Lorenz '96 model [6] as a multi-scale testbed. The governing equations are:

$$
\begin{aligned}
\dot{X}_{k} & =X_{k-1}\left(X_{k+1}-X_{k-2}\right)-X_{k}+F+B_{k} \\
\dot{Y}_{j, k} & =\frac{1}{\varepsilon}\left[Y_{j+1, k}\left(Y_{j-1, k}-Y_{j+2, k}\right)-Y_{j, k}+h_{y} X_{k}\right]
\end{aligned}
$$

with $k=1, \ldots, K$ and $j=1, \ldots, J$. The subgrid term describing the impact of the fast modes onto the slow modes is $B_{k}=\frac{h_{x}}{J} \sum_{j=1}^{J} Y_{j, k}$. By combining a clustering algorithm with local regression fitting [2] a stochastic model of the subgrid term is obtained which is conditional on the state of the resolved variables [4]. The joint probability density of $X_{k}$ and $B_{k}$ is represented as a sum over clusters, each associated with a predictive model:

$$
p\left(X_{k}, B_{k}\right)=\sum_{i=1}^{M} w_{i} p\left(X_{k} \mid i\right) p\left(B_{k} \mid X_{k}, i\right)
$$

The conditional predictive distribution is given as a weighted sum of the individual predictive models

$$
p\left(B_{k} \mid X_{k}\right)=\sum_{i=1}^{M} g_{i}\left(X_{k}\right) p\left(B_{k} \mid X_{k}, i\right)
$$

where the state-dependent weights $g_{i}\left(X_{k}\right)$ are given as the posterior probabilities of associating the state $X_{k}$ with model $i$. Extensions of the scheme are possible, e. g., regression or conditioning also on past values to capture serial correlation or more complex dependences.

Secondly, stochastic reduced-order models of extratropical atmospheric lowfrequency variability are constructed [5]. A quasi-geostrophic (QG) three-level atmospheric model with realistic mean state, variability and teleconnection patterns is used as dynamical framework. The equations of motion are projected 
onto a limited number of leading empirical orthogonal functions (EOFs). Then a regime-weighted model of the tendency error conditional on the state of the resolved modes is estimated from data similar to the one described above for the Lorenz '96 system and added to the bare truncation. A reduced model based on 15 EOFs is able to self-consistently model important statistical and dynamical properties of the low-frequency variability of the QG model. Monitored quantities include the mean state, the variability pattern, momentum fluxes, probability density functions, autocorrelation functions and nonlinear regime behaviour. The present scheme compares favourably with other recently proposed stochastic mode reduction schemes $[1,3]$.

\section{REFERENCES}

[1] C. Franzke, A. J. Majda, Low-order stochastic mode reduction for a prototype atmospheric GCM, Journal of the Atmospheric Sciences 63 (2006), 457-479.

[2] N. A. Gershenfeld, B. Schoner, E. Metois, Cluster-weighted modelling for time series prediction and characterization, Nature 397 (1999), 329-332.

[3] D. Kondrashov, S. Kravtsov, M. Ghil, Empirical mode reduction in a model of extratropical low-frequency variability, Journal of the Atmospheric Sciences 63 (2006), 1859-1877.

[4] F. Kwasniok, Stochastic subgrid-scale parametrization using cluster-weighted modelling, submitted.

[5] F. Kwasniok, Nonlinear stochastic low-order models of atmospheric low-frequency variability using an empirical regime-weighted closure scheme, submitted.

[6] E. N. Lorenz, Predictability - a problem partly solved, in Predictability, ECMWF, Seminar Proceedings (1996), 1-18.

\section{Investigation of latent heat effects at the stratocumulus top using direct numerical simulations Juan Pedro Mellado \\ (joint work with Bjorn Stevens, Heiko Schmidt, Norbert Peters)}

The marine stratocumulus-topped boundary layer plays a fundamental role in the planet radiative energy balance because of its contribution to the earth's albedo. The mixed layer theory from Lilly [1] already identified the relevant parameters several decades ago, pointing to the entrainment rate at the top of the boundary layer as a determining quantity. A complete understanding of the physics of the boundary layer top, however, is still missing, which translates into a variability of order 1 in current models [2].

The cloud-top mixing layer has been used as an idealized configuration designed to investigate particular questions related to the local dynamics of the cloud boundary over length scales of the order of meters and under controlled conditions using direct numerical simulation.

Buoyancy reversal due to evaporative cooling has been considered in the first place. At the stratocumulus top, the relatively warm and dry air current descending from the upper troposphere meets the convection boundary layer and forms a strong inversion. When a parcel of cool fluid from the cloud mixes with the 
upper subsiding layer, molecular transfer of heat tends to warm the former and, at the same time, mass diffusion promotes droplet evaporation, which tends to cool the resulting mixture. When this second mechanism dominates, the final mixture acquires a buoyancy smaller than that of the local environment and tends to sink, setting the fluid into motion. The implications of such processes for the large-scale behavior of the stratocumulus-topped boundary layer, like the observed cloud break-up [3, 4], have been debated for a long time.

A simplified formulation based on a mixture fraction variable $\chi$ has been employed [5]. Physically, the mixture fraction is equal to the relative amount of matter in the fluid particle that proceeds from one of two differentiated regions in the system, and it appears naturally in the limit of very small droplets as a normalized conserved scalar measuring conserved properties, such as the total-water content and the total enthalpy at the cloud boundary. The major assumptions (and thus limitations) of this approach are: (1) the liquid phase can be described as a continuum, (2) local thermodynamic equilibrium exists, and (3) the liquidphase diffusivity is equal to that of vapor and dry air. The main parameter of the system so defined is the non-dimensional ratio $D=-b_{s} / b_{1}$ between the minimum buoyancy anomaly (relative to the lower layer) of the intermediate mixtures, $b_{s}$, and the buoyancy difference across the inversion, $b_{1}$.

The linear stability analysis shows that, if $D>0$, there is an unstable mode with a characteristic time $\sqrt{4 \pi \lambda /\left|b_{s}\right|}$, where $\lambda$ is the wavelength of the perturbation, in addition to interfacial gravity waves with a phase velocity $\sqrt{\lambda b_{1} /(4 \pi)}$ - the system is unstable to small disturbances and there exists a route to turbulence [6]. This instability is the so-called buoyancy reversal instability and the condition $D>0$ is the non-dimensional Randall-Deardorff criterion [3, 4].

However, in the usual atmospheric conditions, the subsequent turbulent motion is too weak to break the inversion and to create cloud holes; the turbulent motion is restricted to the cloud. The argument is that the time scale associated with the restoring force of the inversion and the time scale of the unstable downdraft are in a ratio equal to $\sqrt{D}$ and $D$ is a small number, i.e. the inversion returns to the equilibrium position fast compared to the time that the heavy mixture below needs to move downwards a distance $\lambda$. Results from direct numerical simulations confirm this conjecture [7]. The entrainment rate and turbulent fluctuations caused purely by latent heat effects are about one order of magnitude smaller than the measurements, suggesting that buoyancy reversal due to evaporative cooling alone is not the driving mechanism in cloud-top entrainment.

These simulations also show that molecular processes at the inversion base determine the evolution of the whole system, which helps to explain the difficulties encountered in the past using large-eddy simulation, and highlights the potential of direct numerical simulations as a tool to study some specific problems.

Questions about the cloud-top that remain to be addressed are, for instance: what is the role of local mean shear, finite evaporation rates, settling velocities or preferential clustering of droplets? Can there still be a non-linear coupling between buoyancy reversal and other external forcings, like radiation? 
Financial support by the DFG, SPP 1276 Metström, is gratefully acknowledged. Computational time was provided by the DKRZ in Hamburg.

\section{REFERENCES}

[1] D. K. Lilly, Models of cloud-topped mixed layers under strong inversion, Q. J. Roy. Meteorol. Soc. 94 (1968), 292-309.

[2] B. Stevens, Entrainment in stratocumulus-topped mixed layers, Q. J. Roy. Meteorol. Soc. 128 (2002), 2663-2690.

[3] D. A. Randall, Conditional instability of the first kind upside-down, J. Atmos. Sci. 37 (1980), $125-130$.

[4] J. W. Deardorff, Cloud top entrainment instability, J. Atmos. Sci. 37 (1980), 131-147.

[5] J. P. Mellado, B. Stevens, H. Schmidt, and N. Peters, Two-fluid formulation of the cloud-top mixing layer for direct numerical simulation, Theor. Comput. Fluid Dyn. (2010), in press.

[6] J. P. Mellado, B. Stevens, H. Schmidt, and N. Peters, Buoyancy reversal in cloud-top mixing layers, Q. J. Roy. Meteorol. Soc. 135 (2009), 963-978.

[7] J. P. Mellado, The evaporatively driven cloud-top mixing layer, J. Fluid Mech. (2010), in press.

\section{An adaptive discontinuous Galerkin method for modelling cumulus clouds} ANDREAS MÜLlER

(joint work with Jörn Behrens, Francis X. Giraldo, Volkmar Wirth)

Theoretical understanding and numerical modeling of atmospheric moist convection still pose great challenges to meteorological research. The present work addresses the following question: How important is mixing between cloudy and environmental air for the development of a cumulus cloud? A Direct Numerical Simulation of a single cloud is way beyond the capacity of today's computing power. The use of a Large Eddy Simulation in combination with semi-implicit time-integration and adaptive techniques offers a significant reduction of complexity.

So far this work is restricted to two-dimensional geometry. The compressible Navier-Stokes equations are discretized using a discontinuous Galerkin method introduced by Giraldo and Warburton in 2008 [1]. Time integration is done by a semi-implicit backward difference $[2,3]$. For the first time we combine these numerical methods with an h-adaptive grid refinement. This refinement of our triangular grid is implemented with the function library AMATOS and uses a space filling curve approach [4].

Validation through different test cases shows very good agreement between the current results [5] and those from the literature. For comparing different adaptivity setups we developed a new qualitative error measure for the simulation of warm air bubbles. With the help of this criterion we show that the simulation of a rising warm air bubble on a locally refined grid can be more than six times faster than a similar computation on a uniform mesh with the same accuracy. 


\section{REFERENCES}

[1] F. Giraldo and T. Warburton, A high-order triangular discontinuous Galerkin oceanic shallow water model, Int. J. Numer. Methods Fl. 56 (2008), 899-925.

[2] M. Restelli and F. Giraldo, A conservative discontinuous Galerkin semi-implicit formulation for the Navier-Stokes equations in nonhydrostatic mesoscale modeling, Journal of Scientific Computing 31 (2009), 2231-2257.

[3] F. Giraldo and M. Restelli, High-order semi-implicit time-integrators for a triangular discontinuous Galerkin oceanic shallow water model, Int. J. Numer. Methods Fl. 63 (2010), 1077-1102.

[4] J. Behrens, N. Rakowsky, W. Hiller, D. Handorf, M. Läuter, J. Päpke, K. Dethloff, amatos: Parallel adaptive mesh generator for atmospheric and oceanic simulation, Ocean Modelling 10 (2005), 171-183.

[5] A. Müller, J. Behrens, F. X. Giraldo and V. Wirth, An adaptive discontinuous Galerkin model for modeling cumulus clouds, Proceedings of the V European Conference on Computational Fluid Dynamics ECCOMAS CFD 2010, ISBN: 978-989-96778-1-4.

\section{A potential vorticity dynamics for rotating shallow water on the sphere}

DAvid J. Muraki

(joint work with Andrea Blazenko, Kevin Mitchell \& Chris Snyder)

The standard theory for weather systems in the midlatitude atmosphere is quasigeostrophy (QG), in which the slow, synoptic-scale airflow is described through the advection of potential vorticity (PV). The mathematics of QG is often justified by a limit of zero Rossby number. But, this assumed limit is made invalid across the equator by the vanishing of the Coriolis effect.

A globally-valid analog of QG (sPV), that is based upon the dynamics of PV, is developed for rotating shallow water $(\mathrm{rSW})$ on the sphere. Specifically, a PVstreamfunction relationship is defined which determines the flow velocities for the entire sphere. At midlatitudes, the fluid dynamics are asymptotically equivalent to the beta-plane theory of QG, in the usual small Rossby number sense. In the equatorial regions, wave propagation at short-scales mimics the dispersion relation for equatorial beta waves. Global Rossby waves, as described in recent works by Verkley $(2009,2010)$ and Schubert $(2009)$, are also included within this sPV framework. The sPV model is an extension, to the entire sphere, of the hemispherical PV inversion of McIntyre \& Norton (1999) which imposed a dynamical symmetry across the equator. In addition, the current work includes a large-scale jet so that as a benchmark test of the dynamics, the propagation of waves in the sPV model are shown to be an excellent approximation to computations of the equatorial crossing of topographic waves by Grose \& Hoskins (1979). Finally, the mathematical consistency of these sPV dynamics is demonstrated by the result that the ray theory for the sPV equations is exactly the same as that for the slow modes of the rSW primitive equations.

The computations of the sPV model are done using a double-Fourier spectral method that is efficient, yet accessible to novice modellers of PDEs on the sphere. 
The approach is a modernization of ideas originating from Merilees (1973) and Orszag (1974).

Combining the midlatitude and equatorial wave dynamics into a unified model has been a long-standing conundrum. This work represents a globally-valid asymptotic theory for short-wave dynamics. However, there remains the open question of the significant errors seen in the longest waves in the equatorial region. This is unfortunate for the subgroup of this conference who focus upon the waves of equatorial tropics. Initial discussions with participants, Joseph Biello and Sam Stechmann, seem to suggest that the missing dynamical ingredients near the equator are those captured by the equations of equatorial long-wave theory (Majda/Biello 2002, Schubert 2010). Should this connection prove correct, this represents a significant clue to the form of possible extensions leading to a global wave theory having validity across all scales.
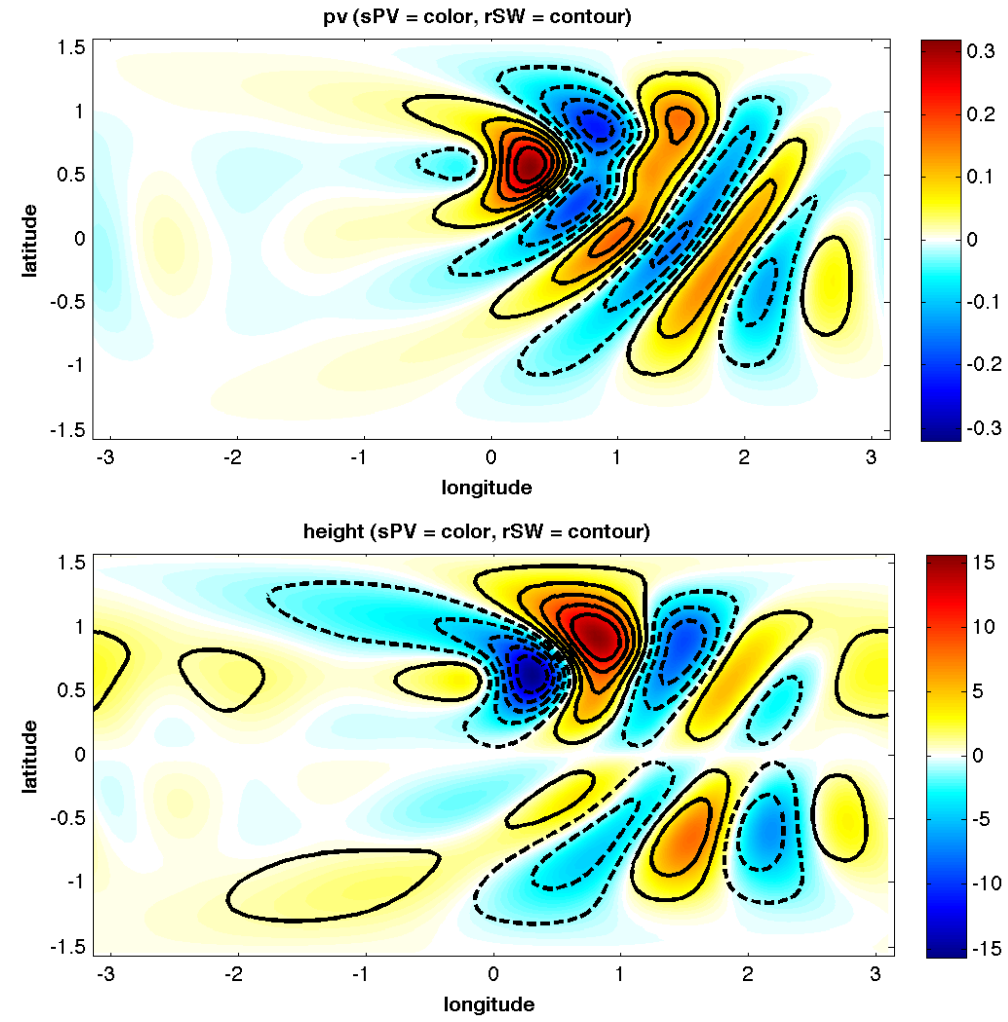

FiguRE 1. The equatorial crossing of topographic waves within the sPV model: disturbance PV, and the associated disturbance height. The flow is similar to that used in the study of Grose \& Hoskins (1979) - super-rotation flow past an isolated mountain centered at $30^{\circ} \mathrm{N}$. 


\section{REFERENCES}

[1] W.T.M. Verkley, A balanced approximation of the one-layer shallow-water equations on a sphere, J. Atmos. Sci., 66 (2009), 1735-1748.

[2] W.T.M. Verkley \& I.R. van der Velde, Balanced dynamics in the tropics, Q.J.R. Meteorol. Soc., 136 (2010), 41-49.

[3] W. Schubert, R.K. Taft \& L.G. Silvers, Shallow Water Quasi-Geostrophic Theory on the Sphere, J. Adv. Model. Earth Syst., 1 (2009), 17pp.

[4] M.E. McIntyre \& W.A. Norton, Potential Vorticity Inversion on a Hemisphere, J. Atmos. Sci., 57 (2000), 1214-1235

[5] P.E. Merilees, The pseudospectral approximation applied to the shallow water equations on a sphere, Atmosphere, 11 (1973).

[6] S.A. Orszag, Fourier series on spheres, Mon. Weather Rev., 102 (1974).

[7] A.J. Majda, \& J.A. Biello, The Nonlinear Interaction of Barotropic and Equatorial Baroclinic Rossby Waves, J. Atmos. Sci., 60 (2002), 1809-1821.

[8] W. Schubert, L.G. Silvers, M.T. Masarik \& A.O. Gonzalez, A Filtered Model of Tropical Wave Motions, J. Adv. Model. Earth Syst., 1 (2009), 11pp.

\section{Some exact solutions for a column model of moist convection JOHN NORBURY \\ (joint work with Anthony Lock)}

A radiative-convective column model of moist convection is presented. In this model, a single-column atmosphere of finite depth is driven by heat and moisture sources at sea-level and throughout the body of the column. Moist convection occurs whenever the column atmosphere is not statically stable; if during convection the atmosphere becomes super-saturated, moisture is released and the air is heated. By adopting a Lagrangian view-point, exact equilibrium solutions for vertical profiles of potential temperature and specific humidity are found by means of our choice of radiative cooling and source terms for moisture and latent heat release. Explicit analytical equilibrium solutions, and bounds on the downward equilibrium velocity, are calculated for three cases: a dry atmosphere; a moist atmosphere for which the saturation specific humidity varies only with pseudo-height; and a moist atmosphere for which the saturation specific humidity varies with both pseudo-height and potential temperature. A numerical method for solving the time-dependent problem is also presented. The system evolves in time towards equilibrium in each of the three cases above. Convergence to the analytical solutions is shown.

For a descending parcel, the solution is classical (smooth) and a parcel descends in finite time according to the environmental lapse rate. We also calculate bounds on the rate of subsidence at equilibrium. In addition, we present a numerical model for the time-dependent behaviour of our system. The numerical equilibrium solutions converge to their analytical counterparts.

The structure of the talk is as follows. First, we introduce the radiativeconvective model, and subsequently write it in Lagrangian form. Second, we calculate explicit analytical equilibrium solutions for three different formulae for $q_{\text {sat }}$ (the saturation specific humidity). We consider: (i) a dry atmosphere (specific 
humidity $q \equiv q_{\text {sat }} \equiv 0$ ); (ii) a moist atmosphere where $q_{\text {sat }}$ is a linear function of pseudo-height only; and (iii) a moist atmosphere where $q_{\text {sat }}$ is a linear function of both potential temperature and pseudo-height. Third, we describe the numerical method. Fourth, we calculate explicit expressions for the equilibrium numerical solutions for each of the three formulae for $q_{s a t}$ from before. As the vertical extent of the geometric elements decreases, we recover the analytical solutions. Fifth, we present representative numerical simulations, and show that equilibrium is attained for sufficiently long time and for a suitably chosen time step. Finally, we draw conclusions. This material summarises the pre-print [1].

\section{REFERENCES}

[1] Lock, A. and J. Norbury, Some exact solutions for a column model of moist convection, submitted to J. Atmos. Sci. (2010).

\section{Flow structure and uncertainty estimation of Ekman boundary layer flow over rough surfaces}

ANTONY Z. OWINOH

(joint work with Omar Knio, Rupert Klein)

Ekman [1] provided analytical solutions of approximate boundary layer equations which revealed how frictional stresses impact on ocean currents on a rotating Earth. He predicted that the surface wind direction was at 45 degrees to the isobar assuming constant eddy diffusivity. The velocity profiles actually observed in the Ekman boundary layer indicate significant deviations from these solutions. The aim of talk is to elucidate how the Ekman layer is modified by the surface properties. This is done by solving the Ekman layer equations [2] with the eddy diffusivity parameterised using the mixing length hypothesis [3]. We find that changes in surface roughness can generally enhance momentum transfer, reduce wind speeds and changes in shear stress direction. An empirical relation expressing the surface shear stress as a function of the surface roughness length and geostrophic wind is also presented.

We next investigate the effect of horizontal heterogeneity of the geostrophic wind on the Ekman pumping. From classical theory one would expect the same magnitude of the Ekman pumping provided the magnitudes of the geostrophic vorticity for both cyclonic and anti-cyclonic system are equal but this has proved not to be the case when one considers the mixing length parameterisation to the eddy diffusivity. We further investigate the influence of the characteristics of the underlying surface in a three-level Quasi-geostrophic (QG) model developed by [4]. In the model, the effect of the surface friction is represented via the process of Ekman pumping. Significant differences are noted when we compare the QG model runs based on classical Ekman pumping and those based on the mixing length hypothesis.

These findings naturally lead us to the question: what is the impact of the surface roughness uncertainty on the model outputs such as the wind profiles, the 
surface stress and on the Ekman pumping? We use the polynomial chaos expansion, recently reviewed by [5], to derive the statistics of the boundary layer flow response to surface roughness uncertainty. The polynomial chaos method employs a spectral representation of the response of the solution to changes of the uncertain parameter and was originally developed by $[6]$ as a relative of the Wiener's Homogeneous Chaos theory [7]. Some results are presented that demonstrate the use of non-intrusive polynomial chaos approach [8] to propagate uncertainty in roughness length using the Ekman layer model. Assuming a Gaussian distribution for the roughness length, it is found that the propagation of surface roughness uncertainty to surface stress is not significant. The next step would be to choose other forms of distributions, such as the uniform distribution for the roughness length. Also, since we have a formula that relates the surface shear stress to the surface roughness length and geostrophic wind, we hope to take the advantage of the polynomial chaos approach to construct a complete spectral representation of the surface shear stress as function of surface roughness length and geostrophic wind based on this formula and thereafter, analyse the effect of surface heterogeneity by coupling the polynomial chaos representations with Karhunen-Loève decompositions of the roughness length and geostrophic wind. Finally, the resulting model will be incorporated into the QG model and effects of roughness length heterogeneity on the larger-scale predictions analysed.

\section{REFERENCES}

[1] V. W. Ekman, On the influence of the Earth's rotation on ocean currents, Arch. Math. Astron. Phys., 2 (1905), 1- 52

[2] J. Pedlosky, Geophysical Fluid Dynamics, Springer-Verlag, Berlin / New York, 1987

[3] A. K. Blackadar, The vertical distribution of wind and turbulent exchange in the neutral atmosphere, Journal of Geophysical Research 67 (1962), 3095-3102.

[4] J. Marshall and F. Molteni, Towards a dynamical understanding of planetary-scale flow regimes, Journal of Atmospheric Sciences 50 (1993), 1792-1818

[5] H. N. Najm, Uncertainty quantification and polynomial chaos techniques in computational fluid dynamics, Annual Review of Fluid Mechanics 41 (2009), 35-52

[6] R. G. Ghanem and P. D. Spanos, Stochastic Finite Elements: A Spectral Approach. SpringerVerlag, Berlin / New York, 1991

[7] N. Wiener The homogeneous chaos, Amer. J. Math., 60 (1938), 879-936.

[8] O. M. Knio and O. P. Le Maîetre, Uncertainty propagation in CFD using polynomial chaos decomposition, Fluid Dynamics Research 38 (2006), 616-640.

\section{A generalized Eliassen balanced vortex model EILEEN PÄSCHKE \\ (joint work with Antony Owinoh, Rupert Klein)}

The Eliassen balanced vortex model is an idealized two-dimensional model that was originally derived by Eliassen [1] in order to investigate the dynamical response of an arbitrary axisymmetric vortex in gradient wind regime to axisymmetric sources of heat and angular momentum. The balance of the vortex is disturbed if the sources are acting on the fluid and a secondary circulation in the meridional plane 
develops. Later on, tropical meteorologists discovered that this model could be used to study certain aspects of tropical cyclone development (e.g. [2], [3] and others).

One shortcoming of the Eliassen balanced vortex model is its limitation to studies of purely axisymmetric tropical cyclone like vortices. Real tropical cyclones are however often highly asymmetric so this model may not capture the effects of azimuthal variations in the wind and thermal fields on the vortex intensification.

In this talk we present a generalized Eliassen balanced vortex model valid for strong vortical flow with higher order asymmetric structure. The derivation of the model is based on a mathematical framework named "An unified approach to meteorological modelling" [4] and which uses asymptotic methods to derive simplified meteorological model equations starting from the full three-dimensional compressible flow equations on the rotating earth. Using this framework together with an explicit treatment of a diabatic source term that describes diabatic heating due to moisture conversion processes [5], two sets of asymptotic equations are derived. The first one (Part I) describes the temporal evolution of the leading order vortex flow in response to sources of momentum and heat and which are represented by the horizontal and vertical advection of a higher order wave-number one wind and potential temperature field, respectively. In a pure axisymmetric setting these sources disappear and the equations reduce to the original balance vortex equations as given by Eliassen. The second one (Part II) includes the governing equations that describe the higher order wave-number one wind and thermal fields and thus can be used to determine the sources of heat and momentum occuring in Part I. Moreover it can be shown that the asymmetric wind and thermal fields described by Part II (i) are entirely induced by diabatic effects, and (ii) are influenced non-trivially by the vortex tilt and the vortex motion.

\section{REFERENCES}

[1] A. Eliassen, Slow thermally or frictionally controlled meridional circulation in a circular vortex, Astrophys. Norv. 5 (1952), 19-60.

[2] K. Ooyama, A dynamical model for the study of tropical cyclone development, Geofis. Int. 4 (1964), 187-198.

[3] H. Sundquist, Numerical simulation of the development of tropical cyclones with a ten-level model. Part I., Tellus 22 (1970), 359-390.

[4] R. Klein, An unified approach to meteorlological modelling based on multiple-scales asymptotic., Advances in Geosciences 15 (2008), 22-33.

[5] R. Klein and A.J. Majda, Systematic multiscale models for deep convection on mesoscales., Theor. and Comput. Fluid Dyn. 20 (2006), 525-552. 


\section{Averaging the global atmospheric circulation Olivier Pauluis \\ (joint work with Frédéric Laliberté and Tiffany Shaw)}

Atmospheric motions occur on a wide range of spatial and temporal scales, from the microscopic scale associated with cloud droplets and ice crystals to the planetary scale responsible for the redistribution of energy, momentum and water vapor around the globe. When describing such global circulation, it is common to consider the zonal-mean flow averaged over a long period of time. It is then possible to obtain a streamfunction associated with the mean meridional overturning circulation. However, the results of such analysis depends critically on the choice for the vertical coordinate. For instance, the Eulerian-mean circulation is obtained by averaging the flow on surfaces of constant pressure. It exhibits a three-cell structure in each hemisphere, with the Hadley cell in the tropical and subtropical regions, the Ferrel cell in the midlatitudes, and the polar cell in the polar regions. In contrast, when the flow is averaged on dry isentropes, defined as surface of constant potential temperature, the circulation exhibit a single Equator-to-Pole overturning cell, with high potential air flowing toward the Pole, and low potential temperature air flowing toward the Equator. More recently, [1, 2, 3] have shown that when the circulation is averaged on moist isentropes, defined as surface of constant equivalent potential temperature, the circulation still exhibits a single cell structure, but with a significantly larger mass transport.

Exact computation the circulation on an arbitrary set of iso-surfaces requires a complete 4-dimensional description of the atmosphere. As such extensive dataset is not always readily available, we have developed a new procedure, the Kinetmatic Transformed Eulerian-Mean (KTEM) that makes it possible to approximate the circulation averaged on surface of a constant state variable $\zeta$ using solely information on the mean state of the atmosphere and second order statistics. Our approach can be viewed as a generalization of the Transformed Eulerian method (TEM) $[4,5,6,7,8]$ which has been previously used to approximate the circulation on dry isentropes. The TEM however has an important limitation in that it can only be applied to cases where the vertical profile for $\zeta$ is monotonic. In contrast, KTEM can be applied to any arbitrary variable $\zeta$. Here, we use KTEM to reconstruct the circulation on dry and moist isentropes based on monthly data from the NCEP Reanalysis [9], and show that the KTEM accurately reproduce all the key features of the isentropic circulations obtained from direct computation.

\section{REFERENCES}

[1] A. Czaja, and J. Marshall, The partitioning of poleward heat transport between the atmosphere and ocean, J. Atmos. Sci. 63 (2006), 1498-1511.

[2] O. Pauluis, A. Czaja, and R. Korty, The global atmospheric circulation on moist isentropes, Science 321 (2008) 1075-1078.

[3] O. Pauluis, A. Czaja, and R. Korty, The global atmospheric circulation in moist isentropic coordinates, J. Climate 23 (2010), 3077- 3093. 
[4] D. G. Andrews, and M. E. McIntyre, Planetary waves in horizontal and vertical shear: The generalized Eliassen-Palm relation and the zonal mean acceleration, J. Atmos. Sci. 33 (1976), 2031-2048.

[5] D. G. Andrews, and M. E. McIntyre, An exact theory of nonlinear waves on a Lagrangianmean ow, J. Fluid. Mech. 89 (1978), 609-646.

[6] M. E. McIntyre, An introduction to the generalized lagrangian-mean description of wave, mean-ow interaction, Pure and Applied Geophysics 118 (1980, 152-176.

[7] H. J. Edmon, B. J. Hoskins, and M. E. McIntyre, Eliassen-Palm cross-sections for the troposphere, J. Atmos. Sci. 37 (1980), 2600-2616.

[8] M. N. Juckes, A generalization of the transformed eulerian-mean meridional circulations , Q. J. R. Meteorol. Soc. 127 (2001), 147-160.

[9] E. Kalnay, M. Kanamitsu, M. Kistler, W. Collins, D. Deaven, L. Gandin, M. Iredell, S. Saha, G. White, M. Chelliah, W. Ebisuzaki, W. Higgins, J. Janowiak, K. C. Mo, C. Popolewski, J. Wang, R. Jenne, and D. Joseph, The NCEP/NCAR 40-Year Reanalysis Project, Bull. Amer. Meteor. Soc. 75 (1996), 437-470.

\section{Data assimilation}

Sebastian Reich

(joint work with Kay Bergemann, Georg Gottwald)

Data assimilation is a technique for combining mathematical models of physical systems with measurements in order to estimate either the state of the system or the parameters of the model. For simplicity, we assume that our model is given in form of a differential equation

$$
\dot{\mathbf{x}}=f(\mathbf{x})
$$

with the initial conditions $\mathbf{x}(0)=\mathbf{x}_{0}$ treated as a random variable with given probability density function (PDF) $\pi_{0}$. In the absence of observations the time evolution of $\pi_{0}$ is provided by Liouville's equation

$$
\pi_{t}=-\nabla_{\mathbf{x}} \cdot(\pi \mathbf{u}), \quad \mathbf{u}(\mathbf{x}):=f(\mathbf{x}),
$$

A measurement

$$
\mathbf{y}_{j}=\mathbf{H x}\left(t_{j}\right)+\eta_{j},
$$

at time $t_{j}$ leads to a discontinuous change of the $\operatorname{PDF} \pi$. Here $\eta_{j}$ represents the measurement error, which is a Gaussian distributed random variable with mean zero and variance $\mathbf{R}$. The change in measure is provided by Bayes' theorem, which states that

$$
\pi\left(\mathbf{x}, t_{j}^{+}\right) \propto \pi\left(\mathbf{x}, t_{j}^{-}\right) \times \exp \left(-L\left(\mathbf{x}, \mathbf{y}_{j}\right)\right)
$$

with loglikelihood

$$
L\left(\mathbf{x}, \mathbf{y}_{j}\right)=\frac{1}{2}\left(\mathbf{y}_{j}-\mathbf{H} \mathbf{x}\right)^{T} \mathbf{R}^{-1}\left(\mathbf{y}_{j}-\mathbf{H} \mathbf{x}\right) .
$$

Mathematically, sequential data assimilation is completely characterized by (2) and (4). However, its numerical implementation is not obvious for nonlinear and high-dimensional models (1). 
An exception is provided by the variance minimizing approach of the ensemble Kalman filter (EnKF) technique [4], which replaced the general PDFs in (4) by Gaussian distributions. The extension of EnKFs to more general PDFs is the subject of intense research.

Our own approach to tackle this problem starts from a continuous embedding of (4), which is obtained from the trivial reformulation

$$
\pi\left(\mathbf{x}, t_{j}^{+}\right) \propto \pi\left(\mathbf{x}, t_{j}^{-}\right) \times \prod_{i=1}^{N} \exp \left(-\Delta s L\left(\mathbf{x}, \mathbf{y}_{j}\right)\right),
$$

of (4) with $\Delta s=1 / N$. In the limit $N \rightarrow \infty$, one obtains the evolution equation

$$
\frac{\partial \pi}{\partial s}=-\pi\left(L-\mathrm{E}_{\pi}[L]\right)
$$

in the artificial time-like variable $s \in[0,1][5]$. Here $\mathrm{E}_{\pi}[L]$ denotes the expectation of the $\log$ likelihood function $L$ with respect to the PDF $\pi$. The key idea is now to find a vector field $\mathbf{v}(\mathbf{x}, s)$ such that (7) becomes a continuity equation

$$
\frac{\partial \pi}{\partial s}=-\nabla_{\mathbf{x}} \cdot(\pi \mathbf{v})
$$

similar to Liouville's equation (2) for the differential equation model (1). The obvious requirement is

$$
\nabla_{\mathbf{x}} \cdot(\pi \mathbf{v})=\pi\left(L-\mathrm{E}_{\pi}[L]\right) .
$$

Solutions $\mathbf{v}$ are not uniquely determined. A possible restriction is provided by $\mathbf{v}=\nabla_{\mathbf{x}} \psi$, where $\psi$ is a suitable potential and which provides a link to optimal transportation theory. The sequential data assimilation problem has now been rephrased in form of two continuity equations (2) and (8) for the PDF $\pi$. Both equations can be approximated by standard (Lagrangian) particle methods with the initial particle locations drawn from the initial PDF $\pi_{0}$.

While to above abstract methodology has not yet been applied to the general sequential data assimilation problem, variance minimizing EnKFs can be put into the above framework which provides new insight and improved algorithmic implementations $[1,2,3]$.

\section{REFERENCES}

[1] K. Bergemann, G. Gottwald and S. Reich, Ensemble propagation and continuous matrix factorization algorithms, Quarterly J. Royal Meteorological Society 135 (2009), 1560-1572.

[2] K. Bergemann and S. Reich, A localization technique for ensemble Kalman filters, Quarterly J. Royal Meteorological Society 136 (2010), 701-707.

[3] K. Bergemann and S. Reich, A mollified ensemble Kalman filter, Quarterly J. Royal Meteorological Society 135 (2010), in press.

[4] G. Evensen, Data Assimilation: The Ensemble Kalman Filter, 2nd edition, Springer-Verlag, 2009 .

[5] S. Reich, A dynamical systems framework for data assimilation, technical report, 2010. 


\section{Hybridizable discontinuous Galerkin methods for geophysical applications}

\section{Marco Restelli}

The discontinuous Galerkin (DG) method is an attractive option for the numerical approximation of the flow equations for geophysical problems $[6,11,7]$. An important drawback of this method is however its computational cost, due to the larger number of unknowns compared with more standard continuous finite element formulations as well as with finite volume/finite difference schemes. In this presentation we sketch two possible strategies that allow reducing the computational cost of a linear system arising from a DG spatial discretization. The first strategy exploits an approximate quadrature rule to reformulate the linear system resulting from a discretization using DG finite elements in space and a semi-implicit integrator in time for the compressible Navier-Stokes equation with stratification as an elliptic problem for the sole pressure variable [11]. The second strategy is based on the hybridization of the DG method and is the subject of a series of papers $[1,2,3,4,5,8,9,10]$; in this presentation, it will be discussed considering the steady-state advection-diffusion-reaction model problem, following [4].

\section{REFERENCES}

[1] B. Cockburn and J. Gopalakrishnan, A characterization of hybridized mixed methods for second order elliptic problems, SIAM J. Num. Anal., 42 (2004), 283-301.

[2] B. Cockburn, B. Dong, and J. Guzmán, A superconvergent LDG-hybridizable Galerkin method for second-order elliptic problems, Math. Comp., 77 (2008), 1887-1916.

[3] B. Cockburn, J. Gopalakrishnan, and R. Lazarov, Unified hybridization of discontinuous Galerkin, mixed, and continuous Galerkin methods for second order elliptic problems, SIAM J. Num. Anal., 47 (2009), 1319-1365.

[4] B. Cockburn, B. Dong, J. Guzmán, M. Restelli, and R. Sacco, A hybridizable discontinuous Galerkin method for steady-state convection-diffusion-reaction problems, SIAM J. Sci. Comp., 31 (2009), 3827-3846.

[5] B. Cockburn, J. Guzmán, and H. Y. Wang, Superconvergent discontinuous Galerkin methods for second-order elliptic problems, Math. Comp., 78 (2009), 1-24.

[6] F.X. Giraldo and M. Restelli, A study of spectral element and discontinuous Galerkin methods for the Navier-Stokes equations in nonhydrostatic mesoscale atmospheric modeling: equation sets and test cases, J. Comp. Phys., 227 (2008), 3849-3877.

[7] F.X. Giraldo and M. Restelli, High-order semi-implicit time-integrators for a triangular discontinuous Galerkin oceanic shallow water model, Int. J. Num. Meth. Fluids, 63 (2010), 1077-1102.

[8] N. C. Nguyen, J. Peraire, and B. Cockburn, An implicit high-order hybridizable discontinuous Galerkin method for linear convection-diffusion equations, J. Comp. Phys., 228 (2009), 3232-3254.

[9] N. C. Nguyen, J. Peraire, and B. Cockburn, An implicit high-order hybridizable discontinuous Galerkin method for nonlinear convection-diffusion equations, J. Comp. Phys., 228 (2009), 8841-8855.

[10] N. C. Nguyen, J. Peraire, and B. Cockburn, A hybridizable discontinuous Galerkin method for Stokes flow, Comp. Meth. Appl. Mech. Eng., 199 (2010), 582-597.

[11] M. Restelli and F.X. Giraldo, A conservative discontinuous Galerkin semi-implicit formulation for the Navier-Stokes equations in nonhydrostatic mesoscale modeling, SIAM J. Sci. Comput., 31 (2009), 2231-2257. 


\section{Incompressible Navier-Stokes flows: eigenvalue problems and complex structures}

\section{IAN ROULSTONE}

\section{INTRODUCTION}

There is an extensive literature on the relationship between the velocity gradient tensor $M=\left\{u_{i, j}\right\}$ and the Hessian matrix of the pressure $P=\left\{p_{, i j}\right\}$; see, for example, Gibbon (2008) for a review. We point out some connections between certain eigenvalue problems and the existence of complex structures, thereby extending the results of Roulstone et al. (2009) to the pressure Hessian.

\section{EigenVAlue PROBLEMS}

The incompressible Navier-Stokes equations in two or three dimensions

$$
\frac{\mathrm{D} \boldsymbol{u}}{\mathrm{D} t}+\nabla p=\nu \Delta \boldsymbol{u}, \quad \nabla \cdot \boldsymbol{u}=0,
$$

can be written, upon taking the gradient of the momentum balance, in the form

$$
\frac{\mathrm{D} M}{\mathrm{D} t}+M^{2}+P=D,
$$

where $D=\nu \Delta M$. Henceforth we shall consider inviscid incompressible flows in three dimensions.

The Poisson equation for the pressure can be written

$$
\operatorname{Tr} P=-\operatorname{Tr}\left(M^{2}\right),
$$

and we note that $-\operatorname{Tr}\left(M^{2}\right)=2 Q_{M}$, where $Q_{M}$ denotes the second invariant of the matrix $M$. Equation (3) is the starting point for generalising the notion of elliptic and hyperbolic conditions on flows in two dimensions to almost-complex and real structures in three dimensional Navier-Stokes flows (Roulstone et al. 2009). The key point is that, for incompressible flows, $Q_{M}$ can be expressed in terms of threeforms. We shall now explore such issues with the Hessian matrix of the pressure as our starting point.

\section{Linear algebra of $P$ AND COMPlex Structures}

The first invariant of $P$ is simply its trace, which is $\Delta p$. The second invariant, $Q_{P}$, of $P$ is equal to the sum of the product of pairs of eigenvalues $Q_{P}=\rho_{1} \rho_{2}+$ $\rho_{1} \rho_{3}+\rho_{2} \rho_{3}$. This invariant is given by the sum of the minors of $P$, which in turn can be expressed as the sum of three three-forms.

To see this, let $(l, m, n)=\left(p_{x}, p_{y}, p_{z}\right)$ (the first derivatives of the pressure). Consider $\mathbb{R}^{6}$ spanned by $(x, y, z, l, m, n)$ (also known as the first jet bundle over $\mathbb{R}^{3}, J^{1} \mathbb{R}^{3}$ ) be a symplectic manifold, with symplectic form $\Omega=\mathrm{d} x \wedge \mathrm{d} l+\mathrm{d} y \wedge$ $\mathrm{d} m+\mathrm{d} z \wedge \mathrm{d} n$. Then defining

(4) $\varpi_{p} \equiv f(\boldsymbol{x}, t) \mathrm{d} x \wedge \mathrm{d} y \wedge \mathrm{d} z-(\mathrm{d} l \wedge \mathrm{d} m \wedge \mathrm{d} z+\mathrm{d} l \wedge \mathrm{d} y \wedge \mathrm{d} n+\mathrm{d} x \wedge \mathrm{d} m \wedge \mathrm{d} n)$, 
where $f(\boldsymbol{x}, t)=\rho_{1} \rho_{2}+\rho_{1} \rho_{3}+\rho_{2} \rho_{3}$ (emphasising the dependence of the eigenvalues on space and time), we restrict this three-form to the graph of $\nabla p$. That is,

$$
\left.\varpi_{p}\right|_{\nabla p}=0
$$

is equivalent to

$$
f(\boldsymbol{x}, t)=\left|\begin{array}{ll}
p_{x x} & p_{x y} \\
p_{y x} & p_{y y}
\end{array}\right|+\left|\begin{array}{ll}
p_{x x} & p_{x z} \\
p_{z x} & p_{z z}
\end{array}\right|+\left|\begin{array}{cc}
p_{y y} & p_{y z} \\
p_{z y} & p_{z z}
\end{array}\right| .
$$

Following precisely the same calculation set out in Roulstone et al. (2009), we can show that the Hitchin Pfaffian, $\lambda_{\varpi_{p}}$, of the three-form $\varpi_{p}$, is given by

$$
\lambda_{\varpi_{p}}=-f(\boldsymbol{x}, t) / 4 .
$$

When $\lambda_{\varpi_{p}}<0$ an almost-complex structure exists. This is the case when $f(\boldsymbol{x}, t)>$ 0 , i.e. when $Q_{P}>0$. From (6), this implies

$$
p_{x x} p_{y y}+p_{x x} p_{z z}+p_{y y} p_{z z}>p_{x y}^{2}+p_{x z}^{2}+p_{y z}^{2} \text {. }
$$

\section{Discussion}

Roulstone et al. (2009) show that an almost-complex structure exists on $T^{*} \mathbb{R}^{3}$ when $\Delta p>0$. The first jet bundle, $J^{1} \mathbb{R}^{3}$, is the appropriate space in which to study the Hessian matrix of the pressure (jet bundles are the setting for the study of Monge-Ampère equations using differential forms). The calculation in Section 3 shows how an almost-complex structure is induced on this manifold when the second invariant of $P$ is positive.

It may be possible to investigate complex structures within the semi-geostrophic equations of meteorology in three dimensions by applying Hitchin's construction to the invariants of the 'stability matrix' - a generalisation of the pressure Hessian.

\section{REFERENCES}

[1] J.D. Gibbon, The three-dimensional Euler equations: Where do we stand?, Physica D 237 (2008), 1894-1904.

[2] I. Roulstone, B. Banos, J.D. Gibbon, \& V. Roubtsov, A geometric interpretation of coherent structures in Navier-Stokes flows, Proc. R. Soc. Lond. A 465 (2009), 2015-2021.

\section{A multi-model ensemble method that combines imperfect models through learning \\ FRANK SELTEN \\ (joint work with Leonie van de Berge,Wim Wiegerinck,Gregory Duane)}

Often an ensemble of numerical models is developed to simulate and predict the behavior of real complex systems, like for example models of the climate system. Each typically has its strengths and weaknesses, but all are imperfect representations of the true system. The standard strategy in predicting the behavior of the true system is to combine the outcomes of all available models by some form of 
weighted averaging and arrive at a best guess supplemented with an assessment of the uncertainties.

In this talk we propose a multi-model ensemble method that allows information exchange between the models during the simulation and learn from observed data of the true system how to optimally implement this. The idea is that the ensemble of interacting models form a consensus on the best simulation by combining the strengths of each.

To illustrate and test the proposed method, we use a chaotic, 3 dimensional ODE system, the Lorenz 1963 model. Imperfect models are created by taking three copies of this system with perturbed parameter values. A super-model is created by the introduction of linear connection terms

$$
\begin{aligned}
& \dot{x}_{k}=\sigma_{k}\left(y_{k}-x_{k}\right)+\sum_{j \neq k} C_{k j}^{x}\left(x_{j}-x_{k}\right) \\
& \dot{y}_{k}=x_{k}\left(\rho_{k}-z_{k}\right)-y_{k}+\sum_{j \neq k} C_{k j}^{y}\left(y_{j}-y_{k}\right) \\
& \dot{z}_{k}=x_{k} y_{k}-\beta_{k} z_{k}+\sum_{j \neq k} C_{k j}^{z}\left(z_{j}-z_{k}\right) \quad k=1,2,3,
\end{aligned}
$$

where $k$ indexes the three imperfect models with perturbed parameter values $\sigma_{k}$, $\beta_{k}$ and $\rho_{k}$ and $C_{k j}^{x}, C_{k j}^{y}$ and $C_{k j}^{z}$ are referred to as connection coefficients. The 18 coefficients involved are learned from data that sample the truth. The solution of the super-model is defined as the average of the three models. By the introduction of the connection coefficients we have effectively constructed a new dynamical system of 3 times the dimensionality of the individual models and may expect much richer dynamical behavior.

For the learning we assume that we have a long time series of observations of the truth. We pick initial conditions from this long time series and perform short integrations with the super-model. A cost function, that depends on the vector of connection coefficients $\boldsymbol{C}$, is defined to measure the ability of the super-model to follow the truth. A super-model solution is determined by finding a minimum in the cost function in the 18 dimensional space of $\boldsymbol{C}$

We randomly perturbed the parameters of the Lorenz models with perturbations as large as $30 \%$ of the standard values and were able to find super-model solutions that matched the chaotic solution of the standard model very close and much closer than the combined solutions of the separate imperfect models. While integrating, the interconnected models fall into synchronized behavior. For a given set of imperfect models a multitude of super-model solutions exist that correspond to local minima and stretched valleys in the cost function. The solutions differ in their ability to simulate the truth by various measures. Not always could we find a super-model solution of good quality for a given set of perturbed models, suggesting that there are certain constraints on the imperfections that must be met in order for the method to work. 
We repeated the same procedure for the 3-dimensional Rössler system and the Lorenz 1984 model and arrived at similar conclusions. These illustrative examples suggest that the super-modeling approach is a promising strategy in modeling real complex systems like the climate system. A number of questions remains to be answered:

- Are other forms of the connections more effective?

- How many connections are required?

- Which variables are to be connected and how often?

- How much data is needed for the learning?

- Are there more effective learning strategies?

- How to handle the slow oceanic time scales?

- What if reality falls outside of the model class?

- Does the supermodel also perform well in a changing climate?

- Do balances and conservation laws place restrictions on the connections?

- Can one choose connections on the basis of insight, without learning?

\section{REFERENCES}

[1] L. van den Berge, F.M. Selten, W. Wiegerinck and G. Duane, A multi-model ensemble method that combines imperfect models through learning, Earth System Dynamics, submitted (2010).

The impact of solar thermal tides on the propagation and dissipation of gravity waves

FABIAN SENF

(joint work with Ulrich Achatz)

Gravity waves (GWs) and thermal tides are important phenomena in middleatmosphere dynamics. Breaking GWs have a major impact on the mean circulation in the middle atmosphere (MA) [1]. Due to the limitations in computational power most complex MA circulation models have to incorporate the effect of unresolved GWs via an efficient parametrization [2]. Typically, these are of vertical column type and ignore horizontal and temporal variations in the background fields. However, highly transient tidal perturbations are always present and dominate diurnal variations in the MA through which the GWs propagate. Even in studies of the interaction between GWs and these thermal tides, a possibly important aspect of tidal dynamics, columnar parametrizations of GWs have been applied which do not account for the time dependence of thermal tides.

A ray tracing technique [3] is used to illuminate the impact of horizontal gradients of the background (including the tides) and its time dependence on the propagation and dissipation of GWs. It is shown that tidal transience leads to a modulation of the absolute, or sometimes called ground-based, frequency of slowly propagating GWs. Due to large tidal wind variations in the upper mesosphere most parts of the assumed GW spectrum are slowed down in critical layer type regions. Then, the combined action of horizontal wave number refraction and frequency modulation 
induce changes in the horizontal phase speed which may exceed the initial phase speed by orders of magnitude. The phase speed variations have the tendency to follow the shape of the tidal background wind. This effect leads to less critical layer filtering of GWs and therefore decreased periodic background flow forcing due to momentum flux divergences as compared to a classical vertical column parametrization of instantaneously adjusting GW trains.

\section{REFERENCES}

[1] D. C. Fritts and M. J. Alexander, Gravity wave dynamics and effects in the middle atmosphere, Rev. Geophys. 41(1) (2003), 1003.

[2] C. McLandress, On the importance of gravity waves in the middle atmosphere and their parameterization in general circulation models, J. Atmos. Sol. Terr. Phys. 60 (1998), 13571383.

[3] W. D. Hayes, Kinematic Wave Theory, Proc. Roy. Soc. Lond. A320 (1970), 209-226.

\section{Aspect ratio effects in rotating Boussinesq flows \\ LESLIE SMith}

(joint work with Susan Kurien)

The Boussinesq approximation is a starting point to describe a fairly broad range of oceanic and atmospheric phenomena, depending on the choice of parameters. Despite the fact that geophysical flows are confined to small-aspect-ratio domains, idealized numerical studies of the Boussinesq equations are a mix of calculations performed in domains of unit aspect ratio and small aspect ratio. From this mix, it is difficult to discern aspect ratio effects from other effects of physical and/or numerical choices such as external forcing, grid spacing and effective viscosity. Here we take initial steps toward quantifying aspect ratio effects. Our investigation is a fundamental study in the sense that we address qualitative and quantitative aspects of the rotating Boussinesq equations, rather than attempt to explain a particular geophysical phenomenon. Nonetheless, straightforward extension of the current work may be relevant to atmospheric data for the horizontal spectrum of kinetic energy measured in the upper troposphere at mid-latitudes [1].

A non-dimensional form of the Boussinesq equations is given by

$$
\begin{gathered}
\frac{D_{\delta}}{D t} \mathbf{u}+R o^{-1} \hat{\mathbf{z}} \times \mathbf{u}+\nabla_{\delta} p+\gamma \rho \hat{\mathbf{z}}=R e^{-1} \boldsymbol{\nabla}_{\delta} \cdot \nabla_{\delta} \mathbf{u}, \\
\frac{D_{\delta}}{D t} \rho-\gamma^{-1}(F r \delta)^{-2} \rho w=(\operatorname{Pr} R e)^{-1} \nabla_{\delta} \cdot \nabla_{\delta} \rho, \quad \nabla_{\delta} \cdot \mathbf{u}=0,
\end{gathered}
$$

where horizontal (vertical) distances $x, y(z)$ are scaled by $L(H)$, and $\hat{\mathbf{z}}$ is the direction of stratification as well as the rotation axis. The total density is $\rho_{T}=$ $\rho_{0}-b z+\rho$ with $b$ positive for stable stratification. The Boussinesq approximation assumes $\rho \ll \rho_{0},|b z|$ with background in hydrostatic balance $\rho_{0} g=\partial p_{0} / \partial z$ ( $g$ is the gravitational acceleration). All velocity components $(u, v, w)$ are scaled by a characteristic large-scale velocity $U$, pressure $p$ by $\rho_{0} U^{2}$ and density fluctuations $\rho$ by $B \rho_{0}$ with $B$ constant. The operator $D_{\delta} / D t=\partial_{t}+\mathbf{u} \cdot \nabla_{\delta}, \nabla_{\delta}=\nabla_{h}+\hat{\mathbf{z}} \delta^{-1} \partial_{z}$, 
$\nabla_{h}=\hat{\mathbf{x}} \partial_{x}+\hat{\mathbf{y}} \partial_{y}$ and we have adopted the shorthand notation $\partial_{i}$ for the partial derivative with respect to $i$. Given the buoyancy frequency $N=\left(g b / \rho_{0}\right)^{1 / 2}$ and Coriolis parameter $f$, the non-dimensional parameters are the Rossby number $R o=U /(f L)$, the Froude number $F r=U /(N H)$, the aspect ratio $\delta=H / L$, the Reynolds number $R e=U L / \nu$, the Prandtl number $\nu / \kappa$ and $\gamma=B g L / U^{2}$ with constraint $\gamma=(\delta F r)^{-1}$ for conservation of energy. The latter constraint selects the appropriate non-dimensional coefficient $B$ in terms of the characteristic (imposed) velocity $U$ by the consistency relation $B=U\left(g \rho_{0} / b\right)^{-1 / 2}$.

The inviscid, non-diffusive limit of the Boussinesq equations conserves potential vorticity $q=\left(\boldsymbol{\omega}+R o^{-1} \hat{\mathbf{z}}\right) \cdot \nabla_{\delta} \rho$ following fluid particles:

$$
\frac{D_{\delta}}{D t} q=\frac{D_{\delta}}{D t}\left((R o \delta)^{-1} \partial_{z} \rho-(F r \delta)^{-1} \boldsymbol{\omega} \cdot \hat{\mathbf{z}}+\boldsymbol{\omega} \cdot \boldsymbol{\nabla}_{\delta} \rho\right)=0,
$$

where $\boldsymbol{\omega}$ is the relative vorticity $\boldsymbol{\omega}=\boldsymbol{\nabla}_{\delta} \times \mathbf{u}$. The constant piece of $q\left(R o^{-1} \mathrm{Fr}^{-1}\right)$ has been dropped since it does not contribute to the conservation law(s).

To start a numerical study of aspect ratio effects, we begin with Burger number $B u=N H /(f L)=R o / F r=1$, two aspect $\operatorname{ratios} \delta=1 / 4,1$ and $R o=F r=\epsilon<1$. For consistency with scaling $(u, v, w)$ by the same large-scale velocity $U$, we choose a large-scale, random force equipartitioned between the three velocity components and the density fluctuations. The objective is to reduce the essential parameter space to $(\epsilon, \delta)$. In keeping with this goal, we use hyper-viscosity and hyper-diffusion of order 8 and an isotropic grid such that the largest resolved wavenumbers are the same in all directions. Periodic boundary conditions allow for pseudo-spectral calculations with resolutions up to $2048 \times 2048 \times 512$ Fourier modes for $\delta=1 / 4$ and $640 \times 640 \times 640$ for $\delta=1$.

The asymptotic limit $R o \sim F r=\epsilon \rightarrow 0$ results in the quasi-geopstrophic (QG) equation for nonlinear interactions between the vortical linear eigenmodes, which are decoupled from the wave modes [2]. QG dynamics are characterized by linear potential vorticity $q_{q g}=(\epsilon \delta)^{-1}\left(\partial_{z} \rho-\boldsymbol{\omega} \cdot \hat{\mathbf{z}}\right)$ and small-scale energy spectrum scaling as $E^{q g}(k) \propto k^{-3}$. For $B u=1$ and small enough $\epsilon$, one expects numerical simulations of the full Boussinesq system (1) to yield (i) $q \approx q_{q g}$, and (ii) smallscale, vortical mode spectrum $E^{0}(k)$ scaling as $E^{0}(k) \propto k^{-3}$. Our simulations show the results (i) and (ii) quite clearly for $\epsilon=0.002$ and $\delta=1,1 / 4$. In the regime of small-enough $\epsilon$ where potential vorticity is dominated by its linear piece, aspect ratio effects are manifest only in the wave component of the flow.

For $\delta=1, \epsilon \ll 1$, benchmark numerical simulations [3] established the scaling $E^{ \pm}(k) \propto k^{-1}$ for the wave-mode energy spectrum $E^{ \pm}(k)$. Since $N / f=4$ for $\delta=1 / 4$, the wave component of the flow shows more tendency for horizontal layering than for $N=f$ and $\delta=1$. The wave-mode spectrum $E^{ \pm}(k)$ for $\delta=1 / 4$ is overall much steeper than $k^{-1}$ for all scales smaller than the forced scales, and appears to exhibit dual scaling. The differences in wave-mode structure for $\delta=1$ and $\delta=1 / 4$ result from a change of internal dynamics, and are not a result of the forcing. Ultimately our goal is to understand flow structure and statistics as a function of $B u, F r, R o$ and $\delta$ (here reduced to two parameters with $B u=1$ with 
$F r=R o=\epsilon)$. For relevance to the Nastrom-Gage spectrum of wind velocities, the study could be expanded to include an external force chosen to mimic observations.

\section{REFERENCES}

[1] G. D. Nastrom \& K. S. Gage, A climatology of aircraft wavenumber spectra observed by commercial aircraft, J. Atmos. Sci. 42 (1985), 950-960.

[2] P. F. Embid \& A. J. Majda, Low Froude number limiting dynamics for stably stratified flow with small or finite Rossby numbers, Geophys. Astrophys. Fluid Dynam. 87 (1998), $1-50$.

[3] P. Bartello, Geostrophic adjustment and inverse cascades in rotating stratified turbulence, J. Atmos. Sci. 52 (1995), 4410-4428.

\section{Geostrophic turbulence near rapid changes in stratification SHAFER SMITH \\ (joint work with Elsa Bernard)}

Geostrophic turbulence near rigid upper or lower boundaries is controlled by the forward cascade of buoyancy variance, exhibiting a shallow energy spectrum, secondary roll-up of filaments into small vortices, and a corresponding vorticity PDF with fat tails. The surface quasigeostrophic (SQG) model, which assumes a semiinfinte volume with zero quasigeostrophic potential vorticity (QGPV) and conservation of buoyancy at its surface, provides the simplest setting in which these effects arise. A family of somewhat more complex models that include finite domain depth, non-zero QGPV and forcing by baroclinic instability [1, 2] demonstrate how these effects can arise in more realistic settings, and reveal the competition between flow characteristics driven by interior QGPV anomalies and those driven by surface buoyancy anomalies. In particular, baroclinically-unstable flows can lead to a surface kinetic energy spectrum with a $K^{-3}$ power law over a range of wavenumbers greater than than the first deformation wavenumber, and a flatter $K^{-5 / 3}$ spectrum beyond some transition wavenumber, $K_{t}$, determined by the relative baroclinic forcing of surface and interior modes. In the idealization of the atmospheric tropopause as a rigid surface overlying the baroclinically-unstable troposphere, this may partially explain the observations of Nastrom and Gage [3].

The tropopause is obviously not a rigid surface, which motivates us to investigate geostrophic turbulence near a smooth jump in stratification. The goal is to understand just how sharp a transition must be to yield SQG-like behavior, and what its horizontal and vertical scales are. We consider a family of idealized buoyancy frequency profiles of the form $N(z)=N_{0}+N_{J} \tanh (z / \delta)$, where $N_{0}$ is the average value, $N_{J}$ is the magnitude of the jump across $z=0$ and $\delta$ the vertical scale of the jump (the profile approaches a step function as $\delta \rightarrow 0$ ). With non-constant $N$, and after Fourier-transforming in the horizontal (assuming periodic lateral boundary conditions), the relationship between the QGPV and the streamfunction is a second order eliptic equation with non-constant coefficients, $\mathcal{L} \psi_{\mathbf{K}}=q_{\mathbf{K}}$, with $\mathcal{L} \equiv-K^{2}+\partial_{z} f^{2} / N^{2} \partial_{z}$, where $\mathbf{K}=(k, l)$ is the horizontal 
wave-vector, $K$ is its magnitude, $f$ is the Coriolis paramter, and $q_{\mathbf{K}}$ and $\psi_{\mathbf{K}}$ are the spectral amplitudes of the QGPV and streamfunction, respectively.

Given $q_{\mathbf{K}}(z)$ and assuming decay conditions for $z \rightarrow \pm \infty$, the streamfunction can be found via the integral

$$
\psi_{\mathbf{K}}=\int_{-\infty}^{\infty} q_{\mathbf{K}}(\xi) G(z, \xi) d \xi
$$

where $\mathcal{L} G(x, \xi)=\delta(z-\xi)$ defines the Green's function $G(z, \xi)$ for the operator $\mathcal{L}$. The Green's function is approximated using the WKB method with the small parameter $K / K_{H}$, where $K_{H}=f / N_{0} H$ and $H$ is an imposed vertical scale, which can be taken as the height of the (neglected) boundaries. The result is

$$
G(z, \xi) \approx-\frac{\sqrt{N(z) N(\xi)}}{2 f} K^{-1} e^{ \pm \frac{K}{K_{H}} \frac{z-\xi}{H}}\left(\frac{\cosh z / \delta}{\cosh \xi / \delta}\right)^{ \pm \frac{K}{K_{\delta}}} .
$$

where $K_{\delta} \equiv f /\left(N_{J} \delta\right)$ is a deformation wavenumber associated with the thickness $\delta$ of the transition layer. When $\delta$ is small, the QGPV will be dominated by a spike at $z=0$, due to the derivative of $N^{-2}$ in the operator $\mathcal{L}$; assuming the QGPV to be dominated by this term, and a forward inertial cascade of QGPV variance, we can use the Green's function inversion to predict the spectrum of kinetic energy for all $\mathbf{K}$ and $z$. The computation results in a SQG-like $-5 / 3$ spectrum from the smallest wavenumber (greater than or near $K_{H}$, due to our WKB approximation) up to $K_{\delta}$. At higher wavenumbers, $K<K_{\delta}$, the spectrum reverts to a -3 shape.

The jump in stratification has two effects: it alters the Green's function in the region of the jump, and it produces a peak in PV near the jump. When the Green's function is integrated against this sharp PV distribution, contributions far from the jump $(|z| \gg \delta)$ are supressed, and the kinetic energy spectrum flattens. This occurs for a range of wavenumbers $K_{H}<K<K_{\delta}$. The vertical distribution of the flattened spectrum decays over a distance proportional to $\delta$. Thus, SQG-effects should be expected at jumps in $N$, even when this jump is rather smooth, but only for wavenumbers $K<K_{\delta}$. Numerical simulations of freely-evolving turbulence initialized with a QGPV distribution dominated by the jump in $N$, and with variance concentrated at the largest model scales, verify these predictions.

\section{REFERENCES}

[1] Tulloch, R. T., and K. S. Smith, A theory for the atmospheric energy spectrum: Depth-limited temperature anomalies at the tropopause, Procedings of the National Academy of Sciences, 103 (2006), 14690-14694.

[2] Tulloch, R. T., and K. S. Smith, Quasigeostrophic turbulence with explicit surface dynamics: Application to the atmospheric energy spectrum, Journal of the Atmospheric Sciences, 66 (2009), 450-467.

[3] Nastrom, G. D., and K. S. Gage, A climatology of atmospheric wavenumber spectra of wind and temperature observed by comercial aircraft, Journal of the Atmospheric Sciences, 42 (1985), 950-960. 


\title{
Uncertainty propagation and quantification in numerical ocean simulations using polynomial chaos expansions
}

\author{
ASHWANTH SRINIVASAN
}

(joint work with W. C. Thacker, M. Iskandarani, O. M. Knio)

Polynomial Chaos Expansions, based on Weiner's concept of homogeneous chaos [1], have been developed in the engineering community to propagate uncertainties in model inputs to uncertainties in model outputs[2, 4, 3]. The approach relies on representing uncertain inputs as functions of random variables of known or assumed probability density function, and expressing the model solution as a spectral expansion in terms of suitable orthogonal basis functions associated with the probability density of the inputs. The coefficients of the spectral expansion are then computed by a Galerkin projection that avoids modification to the original code, and that requires only an ensemble calculation at a carefully selected values of the stochastic variable. Here we present a first application of this methodology to a HYCOM simulation of the circulation in the Gulf of Mexico. In this example, we use two stochastic dimensions to parameterize uncertainties in the boundary conditions and expand the output as a Gauss-Hermite polynomial series truncated to 5 th order. The uncertain boundary conditions are propagated and tracked as uncertainties in model outputs with particular emphasis on those associated with the Loop Current. We present the mean and statistics of the solution obtained from the spectral expansions. We also examine convergence of the expansion by checking the impact of retaining more terms. It is suggested that the method is a viable alternative to other methods for exploring the consequences of a small number of important but uncertain inputs. When the output response surface is smooth, the method offers several advantages including an efficient functional representation of output uncertainties using a small number of coefficients and no-apriori limits on the linearity of the model or constraints on the input/output statistics.

\section{REFERENCES}

[1] N. Wiener, The homogeneous chaos, American Journal of Mathematics 60 (1938), 897-936.

[2] R. G. Ghanem and P. D. Spanos, Stochastic Finite Elements: A Spectral Approach, SpringerVerlag, New York, (1991).

[3] O. P. Le Maître and O. M. Knio, Spectral Methods for Uncertainty Quantification, SpringerVerlag, (2010).

[4] D. Xiu and G. E. Karniadakis, Modeling uncertanity in flow simulations via generalized polynomial chaos, Journal of Computational Physics, 187 (2003), 137-167. 


\section{Multiscale models for cumulus cloud dynamics}

SAMUEl N. StechmanN

(joint work with Bjorn Stevens)

Cumulus clouds involve processes on a vast range of scales - including cloud droplets, turbulent mixing, and updrafts and downdrafts - and it is often difficult to determine how processes on different scales interact with each other. In this article, several multi-scale asymptotic models are derived for cumulus cloud dynamics in order to (i) provide a systematic scale analysis on each scale and (ii) clarify the nature of interactions between different scales. In terms of scale analysis, it is shown that shallow cumulus updrafts can be described by balanced dynamics with a balance between source terms and ascent/descent; this is a cloud-scale version of so-called weak-temperature-gradient models. In terms of multi-scale interactions, a model is derived that connects these balanced updrafts to the fluctuations within the balanced updraft envelope. These fluctuations describe parcels and updraft pulses, and this model encompasses some of the multi-scale aspects of entrainment. In addition to this shallow cumulus model, in order to also provide a broad picture of general cumulus dynamics, multi-scale models are also derived for other scales; these include models for parcels and sub-parcel turbulent mixing and models for deep cumulus. Broadly speaking, the differences in the shallow and deep cases convey the notion that shallow cumulus dynamics are parcel-dominated, whereas deep cumulus dynamics are updraft-dominated; this is largely due to the difference in the apparent magnitude of the background temperature stratification. In addition to their use in guiding theory, the multi-scale models also provide a framework for multi-scale numerical simulations. This work is published in [1].

\section{REFERENCES}

[1] S. N. Stechmann and B. Stevens, Multiscale models for cumulus cloud dynamics, J. Atmos. Sci. (2010), in press.

\section{The diurnal cycle and the meridional extent of the tropics EsteBAn G. TABAK \\ (joint work with Paul A. Milewski)}

This talk proposes a mathematical theory explaining the sharp transition between tropics and extra-tropics in terms of the diurnal cycle of thermal forcing by the sun. This transition, at a latitude of 30 degrees, coincides with the outer edge of the Hadley cells, and is marked by a steep jump in the height of the troposphere, from around fifteen kilometers in the tropics to about nine in the mid and high latitudes. The tropics, equatorwards of 30 degrees, are characterized by easterly surface winds -the Trades- and a strong diurnal signal in the wind, pressure and temperature, often marked by regular daily storms in the rainy season. Polewards of 30 degrees, the winds are westerly, and the weather systems have longer spatio-temporal scales. 
This change of behavior can be explained in terms of diurnal waves, created by thermal forcing and trapped equatorwards of 30 degrees by the Coriolis effect [1]. These waves organize the convective activity, leading to more active mixing and vertical transport in the tropics. This can be illustrated in simple mathematical models, presently ranging from forced linear oscillators to nonlinear conservation laws with entraining shock waves, accounting for the entrainment into the troposphere of air from the surface boundary layer.

\section{REFERENCES}

[1] P. A. Milewski, E. G. Tabak, The diurnal cycle and the meridional extent of the tropics, submitted to Physica D (2010).

\section{Coarse-graining of deterministic dynamics via statistical estimation and optimization}

Bruce Turkington

(joint work with Petr Plecháč)

A fundamental problem confronted throughout the atmospheric and oceanic sciences is that of "coarse-graining" or "sub-grid scale parameterization." In many instances the continuum equations that govern the motion of a geophysical system under study are known and trusted, but a full numerical computation of their solutions is neither feasible nor desirable. Broadly speaking, this situation is typical of any "turbulent" dynamical system, meaning one that excites a wide range of scales (or modes) of motion that interact nonlinearly. For such a system, the goal of a simulation becomes to approximate the average motion of its large scales to within a reasonable accuracy without resolving the small-scale fluctuations that interact with them. In this talk we address the general problem of deriving a closed set of reduced equations for the resolved scales of motion from a principle of maximum compatibility to the complete equations governing the dynamical system. To maintain contact with foundational concepts in nonequilibrium statistical mechanics, we focus on deterministic, Hamiltonian dynamics, and we seek a statistical closure in terms of a selected set of resolved variables.

The underlying microscopic dynamics is Hamiltonian on a phase space $\Gamma$, so that for any observable $F: \Gamma \rightarrow R, d F / d t=\{F, H\}$, where $H$ denotes the Hamiltonian, and $\{\cdot, \cdot\}$ is the Poisson bracket. Finitely many selected observables compose the resolved vector $A=\left(A_{1}, \ldots, A_{m}\right): \Gamma \rightarrow R^{m}$. Typically these $A_{k}$ are slow modes of the system, which provide a coarse-grained description of the state of the system. We put no restrictions on the choice of these (independent) resolved variables, but the quality of the resulting model reduction certainly depends on the choice of $A$.

Under the coarse-graining the unresolved variables are relegated to a statistical description, which we represent by a parametric family of probability densities on $\Gamma$ associated with the resolved vector $A$. The simplest statistical model is the exponential family (of maximum entropy states): $\tilde{\rho}(\lambda)=\exp \left[\lambda^{*} A-\phi(\lambda)\right] \rho_{e q}$, 
relative to a fixed equilibrium density $\rho_{e q}$. The parameter vector $\lambda \in R^{m}$ is dual to the expectation, $\langle A \mid \tilde{\rho}(\lambda)\rangle$, which is the instantaneous macrostate.

Our statistical closure is defined by best-fitting these trial probability densities to the Liouville equation. Specifically, we calculate the residual $R=[\partial / \partial t+$ $\{\cdot, H\}] \log \tilde{\rho}(\lambda)$, for any path $\lambda(t), t_{0} \leq t \leq t_{1} ; R$ represents an information loss rate. We minimize a time-integrated, mean-squared norm, $\int_{t_{0}}^{t_{1}}\|R\|_{\epsilon}^{2} d t$, over the time horizon of prediction; a weight factor $\epsilon \in(0,1]$ on the unresolved component of $R$ is the adjustable parameter in our closure. For each terminal time $t_{1}$, the minimizing path determines a best-estimate, $\hat{\lambda}\left(t_{1}\right)$, at time $t_{1}$.

By introducing a value function, $v\left(\lambda_{1}, t_{1}\right)$, for the defining optimization problem, the estimated macrostate, $\hat{\lambda}\left(t_{1}\right)=\arg \min v\left(\lambda_{1}, t_{1}\right)$, can be deduced from the solution of the relevant Hamilton-Jacobi equation. Heuristically, this HamiltonJacobi equation takes the place of a Fokker-Planck equation in a standard stochastic model, while the value function resembles a minus-log-likelihood function. In our approach it is not necessary to interpose a stochastic model between the given deterministic dynamics and the closed reduced equations, since they follow directly from the information-theoretic, best-fit principle.

In practice, it is not feasible to solve the Hamilton-Jacobi equation when $m$ is not very small. For this reason we derive a pair of equations for the best-fit estimate $\hat{\lambda}\left(t_{1}\right)$ and the corresponding Hessian matrix $D^{2} v\left(\hat{\lambda}\left(t_{1}\right), t_{1}\right)$, which quantifies the information content of the estimate. In the near equilibrium regime under the usual linear response approximations, this system closes exactly; far from equilibrium it requires a further closure assumption, which is akin to a quasi-gaussian approximation. The reduced equations then consist of a vector equation for the estimated macrostate and a matrix Riccati-type equation for the Hessian. In fact, they have a format similar to the "state equation" and a "variance equation" of the Kalman-Bucy filter. But, in our statistical closure problem we are filtering an under-resolved deterministic dynamics, not noisy observations.

The main message of this talk is that the construction of closure schemes for turbulent dynamics can be approached systematically by means of statistical estimation. Our theory paper [1] gives a full exposition of this approach to model reduction, while our companion paper [2] exhibits numerical results and comparisons for a standard test problem in statistical physics - the Zwanzig heat bath, in which a heavy particle is coupled to a large number of light particles.

\section{REFERENCES}

[1] B. Turkington and P. Plecháč, Best-fit quasi-equilibrium ensembles: a new approach to statistical closure for underresolved Hamiltonian dynamics, preprint (2010).

[2] P. Plecháč and B. Turkington, Numerical tests of best-fit statistical closure of underresolved Hamiltonian dynamics, preprint (2010). 


\section{Inertia-gravity-wave generation by sheared potential-vorticity anomalies \\ JACQUES VANNESTE \\ (joint work with François Lott and Riwal Plougonven)}

The spontaneous generation of inertia-gravity waves by slowly evolving balanced flows has attracted attention for two main reasons: at a theoretical level, the mechanism reflects the non-existence of an exactly invariant slow manifold for the (inviscid) primitive equations; at a practical level, it represents a potential source of inertia-gravity waves with non-zero phase speeds. In the last few years, a number of numerical simulations have documented spontaneous generation by providing examples of nearly balanced flows, baroclinically unstable flows and vortex dipoles in particular, in which inertia-gravity waves can be detected $[1,2]$. Asymptotic analysis of explicit solutions, on the other hand, suggests that the spontaneous generation is exponentially small in the Rossby number Ro in the limit Ro $\rightarrow 0[3,4]$. This confirms that dynamical-system results obtained for conservative systems with two time scales $[5,6]$ are relevant to fluid models.

One possible mode of spontaneous generation is through the coupling that occurs between balanced motion and inertia-gravity waves in vertically sheared flows $U(z)$. This coupling can be understood by noting that a balanced motion with horizontal scale $k^{-1}$ and frequency $\omega$, localised around some altitude $z=0$ (with $U(0)=0$ ), has a Doppler-shifted frequency $\omega-k U(z)$ which overlaps with the inertia-gravity-wave frequencies at altitudes such that $|\omega-k U(z)| \geq f$.

As a model of this mechanism, we consider the linear response to potentialvorticity perturbations of the form

$$
q(x, y, z, t)=\mathrm{e}^{\mathrm{i}(k x+l y-\omega t)} \delta(z)
$$

in a linear shear flow $U(z)=\Lambda z$. Specifically, we compute the form of other fields, vertical velocity $w$ for instance, which are associated with the potential vorticity (1). The motivation for this computation is twofold: first, by superposition, one can deduce the response of an arbitrary linear solution $q(x, y, z, t)=$ $q_{0}(x-\Lambda z t, y, z)$; second, if there is a horizontal boundary at $z=0,(1)$ can be interpreted as the surface potential temperature $\theta=\exp [\mathrm{i}(k x+l y-\omega t)]$, so that the computation yields the response to a potential-temperature anomaly.

The vertical velocity, regarded as a function of $\zeta=\Lambda k(z-c) / N$, satisfies

(2) $\zeta\left(1-\zeta^{2}\right) W^{\prime \prime}-2(1-\mathrm{i} \gamma \zeta) W^{\prime}-\left(\mathcal{J}^{2} \zeta+2 \mathrm{i} \gamma\right) W=0, \quad$ where $\mathcal{J}=\frac{N \sqrt{1+\gamma^{2}}}{\Lambda}$

and $\gamma=l / k$. The solution can be expressed in terms of hypergeometric functions [7]. More transparent results are obtained in the large-Richardson regime $\mathcal{J} \gg$ 1 (equivalent to a small-Rossby-number regime for this problem) using a WKB approach [8]. This provides expressions for $W$ in four regions (for $\zeta>0$ ): (i) the quasi-geostrophic region $\zeta=O\left(\mathcal{J}^{-1}\right)$, where the solution decays exponentially; (ii) the region $O(1)=\zeta<1$, where the exponential decay is replaced by the faster decay $\exp \left(-\mathcal{J} \sin ^{-1} \zeta\right)$; (iii) the inertial-level region $|\zeta-1|=O\left(\mathcal{J}^{-2}\right)$; and (iv) 
the wave region $\zeta>1$, where the solution takes the form of a propagating inertiagravity wave. Connection formulas relate the amplitude of the solution in each region and give, in particular, the exponentially small amplitude

$$
k \mathcal{J} \mathrm{e}^{-(\mathcal{J}+\gamma) \pi / 2-\kappa c}
$$

in region (iv). This is the amplitude of what can be interpreted as the inertiagravity-wave signature at high altitude of the balanced motion at $z=0$. The strong dependence of (3) on $\gamma=l / k$ implies that this signature is highly anisotropic for potential-vorticity distributions which are isotropic in the $z=0$ plane.

The asymptotic computation indicates that a solution that is purely decaying away from $z=0$ in the quasi-geostrophic region (i) does not satisfy the proper radiating boundary condition for $|z| \gg 1$. The analysis of the Stokes phenomenon that occurs at the inertial level $\zeta=1$ shows that the radiation condition is satisfied provided that the solution decaying in region (i) is accompanied by an exponentially small multiple of its growing counterpart. More precisely, the streamfunction in region (i) takes the form

$$
\psi=A\left(\mathrm{e}^{-\kappa z}+\mathrm{ie}^{-2 \kappa c} \mathrm{e}^{-\mathcal{J} \pi} \cosh (\gamma \pi) \mathrm{e}^{\kappa z}\right) .
$$

From this, and considering the situation of a horizontal boundary at $z=0$, one can derive a relation between $\left.\psi\right|_{z=0}$ and the potential temperature $\theta=\left.\psi_{z}\right|_{z=0}$ that extends the surface quasi-geostrophic relation $\theta=-\left.\kappa \psi\right|_{z=0}$. A generalisation of the surface quasi-geostrophic model that accounts for the (dissipative) effect of wave radiation through a history-dependent term follows. This makes it possible, among other things, to estimate the growth rate of the instability of Eady (or edge) waves studied in [7].

\section{REFERENCES}

[1] R. Plougonven and C. Snyder, Inertia-gravity waves spontaneously excited by jets and fronts. Part I: different baroclinic life cycles, J. Atmos. Sci., 64 (2007), 2502-2520.

[2] C. Snyder, D. Muraki, R. Plougonven and F. Zhang, Inertia-gravity waves generated within a dipole vortex, J. Atmos. Sci., 64 (2007), 4417-4431.

[3] E. I. Ólafsdóttir, A. B. Olde Daalhuis and J. Vanneste, Inertia-gravity-wave radiation by a sheared vortex, J. Fluid Mech., 596 (2008), 169-189.

[4] J. Vanneste, Exponential smallness of inertia-gravity-wave generation at small Rossby number, J. Atmos. Sci., 65 (2008), 1622-1637.

[5] R. S. MacKay, Slow Manifolds, in Energy Localisation and Transfer, Eds. T. Dauxois, A. Litvak-Hinenzon, R. S. MacKay and A. Spanoudaki, World Sci. (2004), 149-192.

[6] J. Vanneste, Asymptotics of a slow manifold, SIAM J. Appl. Dynam. Syst., 7 (2008), 11631190 .

[7] R. Plougonven, D. J. Muraki and C. Snyder, A baroclinic instability that couples balanced motions and gravity waves, J. Atmos. Sci., 62 (2005), 1545-1559.

[8] F. Lott, R. Plougonven and J. Vanneste, Gravity waves generated by sheared potentialvorticity anomalies, J. Atmos. Sci, 67 (2010), 157-170. 


\section{Linear response theory for statistical ensembles in complex systems with time-periodic forcing XIAOMING WANG \\ (joint work with Andrew J. Majda)}

New linear response formulas for unperturbed chaotic (stochastic) complex dynamical systems with time periodic coefficients are developed here. Such time periodic systems arise naturally in climate change studies due to the seasonal cycle. These response formulas are developed through the mathematical interplay between statistical solutions for the time-periodic dynamical systems and the related skew-product system. This interplay is utilized to develop new systematic quasi-Gaussian and adjoint algorithms for calculating the climate response in such time-periodic systems. New linear response formulas are also developed here for general time-dependent statistical ensembles arising in ensemble prediction including the effects of deterministic model errors, initial ensembles, and model noise perturbations simultaneously . An information theoretic perspective is developed in calculating those model perturbations which yield the largest information deficit for the unperturbed system both for climate response and finite ensemble predictions. This is a joint work with Andrew J. Majda [15].

\section{REFERENCES}

[1] R.V. Abramov, R.V. and A.J. Majda, Blended Response Algorithms for Linear FluctuationDissipation for Complex Nonlinear Dynamical Systems, Nonlinearity, 20, Issue 12, 2007, 27932821

[2] R.V. Abramov, R.V. and A.J. Majda, New approximations and tests of linear fluctuationresponse for chaotic nonlinear forced-dissipative dynamical systems, J. Nonlinear Science, 2007, published electronically.

[3] R.V. Abramov, R.V. and A.J. Majda, New Algorithms for Low Frequency Climate Response, J. Atmos. Sci., accepted and in press, 2008.

[4] G. Branstator and J. Frederiksen, The Seasonal Cycle of Interannual Variability and the Dynamical Imprint of the Seasonally Varying Mean State, Journal of the Atmospheric Sciences Volume 60, Issue 13 (July 2003) pp. 1577-1592, DOI: 10.1175/3002.1

[5] J.S. Frederiksen and G. Branstator, Seasonal variability of teleconnection patterns. J. Atmos. Sci., vol. 62, 1346-1365, 2005.

[6] A.S. Gritsun and G. Branstator, Climate Response Using a Three-Dimensional Operator Based on the FluctuationCDissipation Theorem, Journal of the Atmospheric Sciences Volume 64, Issue 7 (July 2007) pp. 2558C2575 DOI: 10.1175/JAS3943.1

[7] A.S. Gritsoun, G. Branstator and V.P. Dymnikov, Construction of the linear response operator of an atmospheric general circulation model to small external forcing, Russian J. Numer. Anal. Math. Moddeling, 17 (2002), 399-416.

[8] A.S. Gritsun, G. Branstator and A.J. Majda, Climate response of linear and quadratic functionals using the fluctuation-dissipation theorem, J. Atmos. Sci., accepted and in press, 2008.

[9] A.S. Gritsoun and V.P. Dymnikov, Barotropic atmosphere response to small external actions: theory and numerical experiments, zv. Russ. Acad. Sci. Atmosphere Ocean Phys. 35 (1999), no.5, 511-525.

[10] C. Leith, Climate response and fluctuation-dissipation. J. Atmos. Sci., vol. 32, 2022-2025, 1975. 
[11] R. Kubo, M. Toda and N. Hashitsume, Statistical physics II: nonequilibrium statistical mechanics, Springer-Verlag, New York, 1985.

[12] A.J. Majda, R. Abramov and M. Grote, Information theory and stochastics for multiscale nonlinear systems, CRM monograph series, American Mathematical Society, 2005.

[13] A.J. Majda, C. Franzke and B. Khouider, An applied mathematics perspective on stochastic modelling for climate. Phil. Trans. R. Soc. A., vol. 366, 2429-2455, 2008.

[14] A.J. Majda and X. Wang, Nonlinear Dynamics and Statistical Theories for Basic Geophysical Flows. Cambridge University Press, 2006.

[15] A. Majda and X. Wang, Linear Response Theory for Statistical Ensembles in Complex Systems with Time-Periodic Forcing, Comm. Math. Sci., 8 no.1 (2010), 145-172.

[16] T.N. Palmer, A nonlinear dynamical perspective on model error: a proposal for non-local stochastic-dynamic parameterizations in weather and climate prediction models. Q. J. R. Meteorol. Soc., vol. 127, 279-304, 2001

[17] H. Risken, The Fokker-Planck Equations, Springer-Verlag, Berlin, 1984.

[18] J.J. Tribbia and D.P. Baumhefner, The reliability of improvements in deterministic shortrange forecasts in the presence of initial state and modelling deficiencies. Mon. Weather Rev., vol. 116, 2276-2288, 1988.

\section{Higher order spatial discretisation methods for non-hydrostatic models of the atmosphere on regular grids}

ANDREAS WILL

(joint work with Jack Ogaja)

In turbulence modeling small stencils, conservation of the integrals of motion and high order of approximation of the mathematical operators for filtering and derivatives could be realised ([2]). Nowadays such methods are going to be developed for state of the art atmospheric LAMs. In the following the behaviour in idealised test cases of different numerical approximations of an incompressible model and the current status of the development of a state of the art model ( COSMO) for $\mathrm{RCM}$ and NWP is presented.

\section{Small Amplitude Disturbance Growth in a LES model}

The small amplitude disturbance growth (one mode only) of the Poiseuille flow is a two dimensional idealised test case suitable for analysing of the phase and of the amplitude error of a substantial part of the numerical approximation of the model.

The Table gives the type of formulation of the nonlinear term (NLT), the order and type of the approximation of the spatial derivatives in $\mathrm{x}, \mathrm{z}$ and in $\mathrm{y}$ direction for the quadratic (nonlinear) and viscous terms and independently for the pressure term and the continuity equation (projection step) of the schemes investigated.

At a rather high $\left(n_{y}=256\right)$ wall normal and low $\left(n_{x}=8\right)$ wall parallel resolution the 4 th order scheme xz4y2p4e generally over-predicts the energy growth. Consistently xz6y4p6e over-predicts the energy growth too. All other schemes under-predict the energy growth. As expected all compact schemes have nearly no phase errors though the discretizations of the projection step are rather different. 


\begin{tabular}{llccc}
\hline Scheme & NLT of & \multicolumn{2}{c}{ quadratic and viscous terms } & $\begin{array}{c}\partial p / \partial q \text { and } \partial u_{q} / \partial q \\
\text { for } q=x \text { and } q=z\end{array}$ \\
& & $\partial / \partial x, \partial / \partial z$ & $\partial / \partial y$ & 2nd expl. \\
\hline \hline xz2y2p2e & divergence & 2nd expl. & 2nd expl. & 4th expl. \\
xz4y2p4e & divergence & 4th expl. & 2nd expl. & 2nd expl. \\
xz6y2p2e & skew-sym. & 6th impl. & 2nd expl. & 4th expl. \\
xz6y2p4e & skew-sym. & 6th impl. & 2nd expl. & 6th expl. \\
xz6y2p6e & skew-sym. & 6th impl. & 2nd expl. & 6th expl. \\
xz6y4p6e & skew-sym. & 6th impl. & 4th expl. & thl \\
\hline
\end{tabular}

TABLE 1. Numerical schemes used. Here $x$ denotes the mean flow and $y$ the wall normal direction. expl. denotes explicit or centr. diff. and impl. implicit or compact scheme. See [1] for details of the compact schemes.

Resolving the wave with 8 points the 2 nd order scheme xz2y2p2e exhibits $10 \%$ and the 4 th order scheme xz4y2p4e $2 \%$ phase error.

The energy growth results are surprising. The compact scheme xz6y4p6e meets perfectly the theoretical energy growth. Interestingly xz6y2p4e and xz6y2p2e provide rather high energy growth errors. The unstable mode became stable. A higher order approximation of the projection step is therefore required to meet small energy growth errors. As shown in [1] the general results are confirmed by turbulent calculations.

\section{2D STATIONARY MOUNTAin FLOW IN THE COSMO MODEL}

The horizontal discretisation schemes available with the 3rd order RK time integration of the non-hydrostatic cosmo_4.11 (and WRF) model are the standard 2nd order central difference scheme with 3rd and 5th upwind and 4th and 6th order centr.diff. discretisation approximations in the horizontal advection term.

We investigated the hydrostatic linear case (HL2DM) of the idealized test case (ITC) 'stationary 2D flow over the mountain' (2DM). The results show clearly that the convergence properties of the 2 nd order central difference scheme are consistent with the theoretical order of convergence. With increasing formal order of the advection scheme the convergence order in the $2 \mathrm{D}$ hydrostatic mountain flow test decreases and reaches 1 for the 5 th and 6 th order schemes, nearly independently on the variable and error norm chosen.

\section{Summary and Conclusions}

The higher order compact scheme xz6y4p6e described by [1] has the desired properties, as shown by small amplitude disturbance growth. The 2nd order scheme $(\mathrm{xz} 2 \mathrm{y} 2 \mathrm{p} 2 \mathrm{e})$ was shown to require a resolution of the wavelength $L_{x}$ by at least 64 points accepting a $1 \%$ error in amplitude and phase. Using a much coarser resolution of $\Delta x=L_{x} / 8$ the higher order compact scheme xz6y4p6e revealed practically perfect results. The phase error was found to be fairly independent of the discretization of the projection step. Explicite and implicite higher 
order discretization of the projection step (required for avoiding of amplitude errors) show the same energy growth. Therefore discretisation of the projection step has not to be of the same kind as for the other terms.

The lack of higher order convergence of schemes with higher order advection terms is consistent with the result found for the turbulence scheme, where the mixing of high order advection and low order pressure gradient showed high order phase error convergence but low order amplitude error convergence with absolute errors much higher for the higher order schemes than for the standard second order scheme. However, the significant decrease of the order of convergence of the higher order advection schemes was surprising and indicates an incosistency.

\section{REFERENCES}

[1] H.-J. Kaltenbach, Phase-Error Reduction in Large-Eddy Simulation Using a Compact Scheme, EUROMECH Colloquium 412 (2002), 83-98.

[2] Y. Morinishi et al., Fully Conservative Higher Order Finite Difference Schemes for Incompressible Flow, J.Comp.P. 143 (1998), 90-124.

[3] M. Sommer and S.Reich, Phase space volume conservation under space and time discretization schemes for the shallow-water equations, in preparation $\mathbf{x x}$ (2010), 1-7.

\section{A simple method to improve the time-stepping in atmosphere and ocean models}

\section{Paul D. Williams}

From a functional perspective, the task of predicting future weather and climate may be reduced to the following iterative procedure. First, given the state of the atmosphere, ocean, and other Earth-system components at any time (the input), use the governing equations to compute the state at a slightly later time (the output). Then, repeat the loop as many times as required, always using the previous output as the next input.

The above prediction framework presents three main challenges, each of which potentially degrades the reliability of the forecast. First, Earth observations, which always contain measurement errors, are required to serve as the initial state. Second, the vast array of active physical processes and interactions is incompletely known and imperfectly represented in the spatially truncated governing equations. Third, the discrete stepping from one time level to the next is merely an approximation to the exact time-continuous evolution. This talk presents a possible avenue for progress with the third of these three challenges, which has received scant attention compared to the extensive research efforts devoted to the first two.

A major problem with the widely used leapfrog time-stepping scheme is that it admits spurious computational modes [5, 4, 3]. In general, a differential equation that is first order in time has one degree of freedom, but an $n$-time-level numerical approximation to it constitutes an $(n-1)$ th-order difference equation with $n-1$ degrees of freedom. Of these $n-1$ modes, one is the physical mode and the remaining $n-2$ are computational modes. The leapfrog is a three-time-level scheme, so one computational mode arises in it, in addition to the physical mode, 
because a second-order difference equation is used to approximate a first-order differential equation. The computational mode (or parasitic mode) is manifest as a spurious oscillation between even and odd time steps, which is referred to as time splitting.

The most widely used solution to time splitting is to apply a time filter during the time-stepping procedure. [7] designed such a filter for the leapfrog scheme and [1] showed that it selectively suppresses the computational mode but leaves the physical mode relatively undamped at low frequencies. The filter is now referred to as the Robert filter, the Asselin filter, or the Robert-Asselin filter. The behavior of the filter has been investigated not only for simple equation sets, with no space dependence, but also for the shallow-water equations [6] and the hydrostatic primitive equations [2].

Despite its unquestioned success, the Robert-Asselin-filtered leapfrog scheme suffers from two related problems. First, in addition to suppressing the computational mode, the scheme also weakly suppresses the physical mode. Therefore, physical quantities (e.g., energy) that are conserved by the time-continuous equations are not necessarily conserved by the time-discretized equations when the filter is activated. The damping and nonconservation may be benign for sufficiently short integrations, but possibly not for longer ones. Second, the Robert-Asselin filter severely degrades the leapfrog scheme's numerical accuracy, measured as the rate at which the error tends to zero as the time step is progressively refined.

This talk proposes a simple modification to the Robert-Asselin filter, which does conserve the three-time-level mean state [8]. When used in conjunction with the leapfrog scheme, the modification vastly reduces the impacts on the physical mode and increases the numerical accuracy for amplitude errors by two orders, yielding third-order accuracy. The modified filter could easily be incorporated into existing general circulation models of the atmosphere and ocean. In principle, it should deliver more faithful simulations at almost no additional computational expense.

\section{REFERENCES}

[1] R. Asselin, Frequency filter for time integrations, Monthly Weather Review 100 (1972), $487-490$.

[2] E. Cordero and A. Staniforth, A problem with the Robert-Asselin time filter for three-timelevel semi-implicit semi-Lagrangian discretizations, Monthly Weather Review 132 (2004), 600-610.

[3] D. R. Durran, Numerical Methods for Wave Equations in Geophysical Fluid Dynamics (1999), Springer-Verlag.

[4] G. J. Haltiner and R. T. Williams, Numerical Prediction and Dynamic Meteorology (1980), Wiley.

[5] F. Mesinger and A. Arakawa, Numerical Methods Used in Atmospheric Models. Global Atmospheric Research Program (GARP) Publications Series 17, Vol. I (1976), GARP.

[6] R. Schlesinger, L. Uccellini and D. Johnson, The effects of the Asselin time filter on numerical solutions to the linearized shallow-water wave equations, Monthly Weather Review 111 (1983), 455-467.

[7] A. J. Robert, The integration of a low order spectral form of the primitive meteorological equations, Journal of the Meteorological Society of Japan 44 (1966), 237-245. 
[8] P. D. Williams, A Proposed Modification to the Robert-Asselin Time Filter, Monthly Weather Review 137 (2009), 2538-2546.

\section{Zonation}

WiLliam R. YOUNG

(joint work with Kaushik Srinivasan)

Zonal jets are the result of a poorly understood process via which spontaneously arising Reynolds stresses destroy an initial state of spatially homogeneous $\beta$-plane turbulence. The ultimate equilibration of this instability results in a mature pattern of zonal jets, co-existing with turbulence. All properties of the turbulence, particularly the transport of scalars and potential vorticity, are substantially modified by the jets. I discussed new approaches to quantitatively analyzing the jetforming instability ("zonation") and parameterizing transport of momentum in these flows. The main hypothesis is that the instability is a transfer of energy, non-local in wavenumber, from the forcing scale directly to the jet scale. Preliminary results are supportive of this hypothesis and suggest analytic approaches providing quantitative relations connecting momentum fluxes to mean shear i.e., "negative eddy viscosities".

The problem is to parameterize the eddies flux on the right hand side of the zonal-mean momentum balance,

$$
\bar{u}_{t}+\mu \bar{u}=-\left(\overline{u^{\prime} v^{\prime}}\right)_{y} .
$$

Diagnosis of turbulence simulations shows that although eddies with scale comparable to the zonal jets themselves contain most of the energy, these large and energetic eddies contribute almost nothing to $\overline{u^{\prime} v^{\prime}}$. Instead, small forcing-scale eddies are the main contributors to the Reynolds stress $\overline{u^{\prime} v^{\prime}}$. In other words, zonal jets are driven by a spectrally nonlocal transfer of energy. The Taylor identity,

$$
\overline{v^{\prime} \zeta^{\prime}}=-\left(\overline{u^{\prime} v^{\prime}}\right)_{y}
$$

and the notion of potential-vorticity mixing, suggests a diffusive parameterization in which a positive eddy diffusivity $\kappa_{e}$ relates the eddy flux to the mean gradient of potential vorticity:

$$
-\left(\overline{u^{\prime} v^{\prime}}\right)_{y} \stackrel{?}{=}-\kappa_{e}\left(\beta-\bar{u}_{y y}\right) .
$$

However Thompson \& Stewart (1977) pointed out that this parameterization does not conserve momentum: integrating the relation above over $y$, and assuming no momentum flux through the boundaries, results in the contradiction

$$
0=-\kappa_{e} \beta \int \mathrm{d} y \text {. }
$$

At least in the weakly nonlinear limit this issue is resolved by the rigorous derivation of an amplitude equation (e.g., Manfroi \& Young 1999). This description, 
which is equivalent to the Cahn-Hilliard equation

$$
\bar{u}_{t}+\mu \bar{u}=-\left(\nu \bar{u}+\eta \bar{u}_{y y}+\alpha_{2} \bar{u}^{2}-\alpha_{3} \bar{u}^{3}\right)_{y y} .
$$

is consistent with momentum conservation and all symmetries of the equations of motion. The underlying variational structure of the Cahn-Hilliard equation explains the observed stability of zonal jets and solutions of the Cahn-Hilliard equation resemble observed jet profiles. These facts support the hypothesis that the Cahn-Hilliard description applies well outside of the weakly-nonlinear limit in which it is derived.

\section{REFERENCES}

[1] Thompson, R.E. and Stewart, R.W., The balance and redistribution of potential vorticity within the ocean. Dynamics of Atmospheres and Oceans, 1, (1977) 299-321.

[2] Manfroi, A.J., \& Young, W.R., Slow evolution of zonal jets on the beta-plane. Journal of the Atmospheric Sciences, 56, (1999) 784-800.

\section{Instabilities of buoyancy driven coastal currents and their nonlinear evolution in the two-layer rotating shallow water model \\ VLADIMIR ZEITLIN}

(joint work with François Bouchut, Jonathan Gula)

We investigate linear and nonlinear stability of buoyancy-driven coastal currents in the two-layer rotating shallow water model. The coastal current is modelled by a layer of lighter fluid of variable depth and variable along-shore velocity, the two being in geostrophic balance, extending from the straight vertical border towards the outcropping density front where it terminates. It is superimposed over the heavier fluid at rest extending down to the flat bottom, and out from the coast to infinity. We accomplish an exhaustive linear stability analysis of such configuration with the help of spectral collocation method [1]. Linear instabilities are identified and classified according to the physical nature of the resonating waves which form the unstable modes. The dependence of the instabilities of the depth and/or density ratio of the layers is investigated.

In order to understand the nonlinear stage of instability, fully nonlinear direct numerical simulations (DNS) using a novel high-resolution entropy-satisfying well-balanced finite-volume scheme of [2], which copes with losses of hyperbolicity associated to shear (Kelvin-Helmholtz type) instabilities in multi-layer systems, were performed. The simulations were initialized with different unstable modes identified by the linear stability analysis, following the approach used earlier to study outcropping fronts within the one-layer shallow water model [3].

The main conclusions following from the DNS are as follows. In the case of a barotropically stable upper-layer flow (i.e. a flow which is stable in the limit of infinitely deep lower layer), for deep, but not excessively deep lower layers the system is destabilized by an instability due to the resonance between a lower-layer Rossby wave, and an upper-layer frontal wave propagating along the outcropping 
line (RF instablity). The growing RF instability leads to breaking of the Rossby and the frontal wave in respective layers, and formation of coherent vortices which are able to detach from the coast due to their dipolar structure in the lower layer. Kelvin front formation at the coast, due to the breaking of non-dispersive Kelvin waves, plays an important role in this process. For sufficiently shallow lower layers the short-wave shear instabilities dominate the initial evolution of the flow. They are, however, rapidly smeared out by dissipation, and do not lead to substantial reorganization of the flow, which is then subject to the RF instability with similar, as described above, evolution.

In the case of barotropically unstable upper-layer flow, and sufficiently deep lower layers, the instability resulting from the resonance of the frontal and Kelvin waves in the upper layer (KF instability), dominates. However, at the later stages of the evolution the baroclinic RF instability steps in and excites vortex structures in the lower layer. The whole system then undergoes baroclinic instability leading to coherent vortices appearing in both layers and eventually detaching from the coast. For shallower lower layers, again, the shear instabilities dominate at initial stages, being eliminated then by dissipation. They do not much influence the development of the upper layer KF instability, and subsequent switching of slower RF baroclinic instability. This latter leads to the formation of coherent vortices with pronounced baroclinic structure.

\section{REFERENCES}

[1] L.N. Trefethen, "Spectral methods in MATLAB", SIAM (2000).

[2] F. Bouchut and V. Zeitlin, A robust well-balanced scheme for multi-layer shallow water equations, Discr. Cont. Dyn. Sys., ser. B, 13 (2010), 739-758.

[3] E. Scherer and V. Zeitlin, Instability of coupled geostrophic density fronts and its nonlinear evolution, J. Fluid Mech., 613 (2008), 309-327. 


\section{Participants}

\section{Dr. Ulrich Achatz}

Johann Wolfgang Goethe Universität

Fachbereich

Geowis-

senschaften/Geographie

Altenhöferallee 1

60438 Frankfurt

\section{Tommaso Benacchio}

Vicolo Cigolo 14b

I-35123 Padova

Prof. Dr. Joseph Biello

Department of Mathematics

University of California, Davis

1, Shields Avenue

Davis , CA 95616-8633

USA

\section{Dr. Simona Bordoni}

California Institute of Technology

Environmental Sciences \& Engineering

Pasadena, CA 91125

USA

Prof. Dr. Yann Brenier

Laboratoire J.-A. Dieudonne

UMR CNRS 6621

Universite de Nice Sophia-Antipolis

Parc Valrose

F-06108 Nice Cedex 2

Prof. Dr. Oliver Bühler

Center for Atmosphere Ocean Science

Courant Institute of Math. Sc.

New York University

251 Mercer Street

New York, NY 10012-1110

USA

\section{Prof. Dr. Paola Cessi}

Scripps Institution for

Oceanography, Physical Oceanography

University of California San Diego

9500 Gilam Drive

La Jolla CA 92093-0230

USA

Dr. George Craig

Institut für Theoretische

Meteorologie

Universität München

Theresienstraße 39

80333 München

Prof. Dr. Stamen Dolaptchiev

Johann Wolfgang Goethe Universität

Fachbereich

Geowis-

senschaften/Geographie

Altenhöferallee 1

60438 Frankfurt

Prof. Dr. Joseph Egger

Institut für Theoretische

Meteorologie

Universität München

Theresienstraße 39

80333 München

\section{Dr. Jason Frank}

Centrum Wiskunde \& Informatica (CWI)

Postbus 94079

NL-1090 GB Amsterdam

Dr. Christian Franzke

Natural Environment Research Council

British Antarctic Survey

High Cross, Madingley Road

GB-Cambridge, CB3 OET 


\section{Dr. Dargan M. Frierson}

Atmospheric Science and Applied

Mathematics

University of Washington

Box 351640

Seattle WA 98195-1640

USA

\section{Dr. Ed Gerber}

Center for Atmosphere Ocean Science Courant Institute of Math. Sc.

New York University

251 Mercer Street

New York, NY 10012-1110

USA

\section{Dr. Dimitris Giannakis}

New York University

Courant Institute of Mathematical

Sciences

251 Mercer Street

New York , NY 10012

USA

\section{Prof. Dr. Francis Giraldo}

Dept. of Applied Mathematics

Naval Postgraduate School

Monterey , CA 93943

USA

\section{Dr. Andrey Gritsun}

Institute of Numerical Mathematics of the Russian Academy of Sciences ul. Gubkina 8

Moscow 119333

RUSSIA

Prof. Dr. Marcus Grote

Mathematisches Institut

Universität Basel

Rheinsprung 21

CH-4051 Basel
Prof. Dr. Illia Horenko

Universita della Svizzera Italiana

Facolta di Scienze Informatiche

Via Giuseppe Buffi 13

CH-6904 Lugano

\section{Prof. Dr. Boualem Khouider}

Dept. of Mathematics \& Statistics University of Victoria

P.O.Box 3060

Victoria, BC V8W 3R4

CANADA

\section{Prof. Dr. Rupert Klein}

Fachbereich Mathematik \& Informatik Freie Universität Berlin

Arnimallee 6

14195 Berlin

\section{Dr. Peter Korn}

Max Planck Institut für Meteorologie Universität Hamburg

Bundesstr. 55

20146 Hamburg

Dr. Frank Kwasniok

College of Engineering, Mathematics and Physical Sciences

Harrison Building

North Park Road

GB-Exeter EX4 4QF

\section{Dr. Juan-Pedro Mellado}

Max-Planck-Institut für

Meteorologie

Universität Hamburg

Bundesstr. 53

20146 Hamburg

Andreas Müller

Institut für Physik der Atmosphäre

Universität Mainz

Becherweg 21

55099 Mainz 
Prof. Dr. David James Muraki

Department of Mathematics

Simon Fraser University

8888 University Dr.

Burnaby , B.C. V5A 1S6

CANADA

\section{Prof. Dr. John Norbury}

Mathematical Institute

Oxford University

24-29 St. Giles

GB-Oxford OX1 3LB

\section{Dr. Antony Z. Owinoh}

Fachbereich Mathematik \& Informatik Freie Universität Berlin

Arnimallee 6

14195 Berlin

\section{Dr. Eileen Paeschke}

Institut für Mathematik

Technische Universität Berlin

Sekr. MA 3-3

Straße des 17. Juni 136

10623 Berlin

Prof. Dr. Olivier Pauluis

Center for Atmosphere Ocean Science

Courant Institute of Math. Sc.

New York University

251 Mercer Street

New York , NY 10012-1110

USA

Prof. Dr. Sebastian Reich

Institut für Mathematik

Universität Potsdam

Am Neuen Palais 10

14469 Potsdam

\section{Prof. Dr. Marco Restelli}

Departamento de Matematicas

Universidad de Sevilla

Tarfia s/n

E-41012 Sevilla
Prof. Dr. Ian Roulstone

Department of Mathematics

University of Surrey

Guildford

GB-Surrey GU2 7XH

\section{Dr. Frank Selten}

Department Global Climate

Royal Netherlands Meteorological

Institute (KNMI)

P.O. Box 201

NL-3730 AE De Bilt

\section{Fabian Senf}

Leibniz-Institut f. Atmosphärenphysik an der Universität Rostock e.V.

Schloßstr. 6

18225 Kühlungsborn

\section{Prof. Dr. Leslie Smith}

Department of Mathematics University of Wisconsin-Madison

480 Lincoln Drive

Madison , WI 53706-1388

USA

Prof. Dr. Shafer Smith

New York University

Courant Institute of Mathematical

Sciences

251 Mercer Street

New York, NY 10012

USA

Dr. Ashwanth Srinivasan

Division of Meteorology and Physical

Oceanography

4600 Rickenbacher Causeway

Miami , FL 33149

USA 
Prof. Dr. Samuel N. Stechmann

University of California

Department of Mathematics

520 Portola Plaza

Los Angeles , CA 90095-1555

USA

\section{Prof. Dr. Bjorn Stevens}

Max-Planck-Institut für

Meteorologie

Universität Hamburg

Bundesstr. 53

20146 Hamburg

\section{Prof. Dr. Esteban G. Tabak}

Courant Institute of

Mathematical Sciences

New York University

251, Mercer Street

New York, NY 10012-1110

USA

Prof. Dr. Edriss S. Titi

Department of Mathematics University of California, Irvine Irvine, CA 92697-3875

USA

Prof. Dr. Bruce Turkington

Dept. of Mathematics \& Statistics University of Massachusetts

Amherst MA 01003-4515

USA

Dr. Jacques Vanneste

School of Mathematics

University of Edinburgh

James Clerk Maxwell Bldg.

King's Buildings, Mayfield Road

GB-Edinburgh EH9 3JZ
Prof. Dr. Xiaoming Wang

Department of Mathematics

Florida State University

Tallahassee , FL 32306-4510

USA

Dr. Andreas Will

BTU Cottbus

LS Umweltmeteorologie

Burger Chaussee 2, Haus 4/3

03044 Cottbus

\section{Dr. Paul Williams}

Department of Meteorology

University of Reading

Earley Gate

PO Box 243

GB-Reading RG6 6BB

Prof. Dr. William R. Young

Scripps Institution for

Oceanography, Physical Oceanography

University of California San Diego

9500 Gilam Drive

La Jolla CA 92093-0230

USA

Prof. Dr. Vladimir Zeitlin

Lab. de Meteorologie Dynamique

Ecole Normale Superieure

24, rue Lhomond

F-75231 Paris Cedex 05 
